\title{
NEW CARTESIAN GRID METHODS FOR INTERFACE PROBLEMS USING THE FINITE ELEMENT FORMULATION
}

\author{
ZHILIN LI*, TAO LIN ${ }^{\dagger}$, AND XIAOHUI WU $\ddagger$
}

\begin{abstract}
New finite element methods based on Cartesian triangulations are presented for two dimensional elliptic interface problems involving discontinuities in the coefficients. The triangulations in these methods do not need to fit the interfaces. The basis functions in these methods are constructed to satisfy the interface jump conditions either exactly or approximately. Both non-conforming and conforming finite element spaces are considered. Corresponding interpolation functions are proved to be second order accurate in the maximum norm. The conforming finite element method has been shown to be convergent. With Cartesian triangulations, these new methods can be used as finite difference methods. Numerical examples are provided to support the methods and the theoretical analysis.
\end{abstract}

Key words. interface problems, Cartesian grid, immersed interface method, finite element method, jump conditions, conforming and non-conforming basis functions, error analysis.

AMS subject classifications. 65L10, 65L60, 65L70.

1. Introduction. In this paper, we develop finite-element immersed interface methods using Cartesian grids for differential equations with discontinuities in the coefficients across one or several arbitrary interfaces in the solution domain. These problems are referred to as interface problems in this paper. A model problem is

$$
\begin{aligned}
-\nabla \cdot(\beta \nabla u) & =f, \quad(x, y) \in \Omega, \\
\left.u\right|_{\partial \Omega} & =0,
\end{aligned}
$$

defined in a domain $\Omega$ with an immersed interface $\Gamma$, see Fig. 1.1 for an illustration. A vast collection of applications involve solving such an equation, for example, the projection method for solving NavierStokes equations involving two phase flow $[4,8,21,44]$, the Hele-Shaw flow $[19,20]$ and many others. If a problem of interest involving two different materials, such as water and air, solid and liquid in solidification problems, the coefficient $\beta$ will typically have a jump across the interface between two materials. In some cases, the jump can be very big, for example, the ratio of the density of the air and water is about 1:1000 in the magnitude.

Our methods can also be applied to those models whose source term $f$ in (1.1) have a delta function singularity, for example

$$
f(\mathbf{x})=f_{c}(\mathbf{x})-\int_{\Gamma} Q(\mathbf{X}(s)) \delta(\mathbf{x}-\mathbf{X}(\mathbf{s})) d s
$$

where $f_{c}$ is a bounded function, $\delta$ is the two dimensional Dirac-delta function, $\mathbf{X}(\mathbf{s})$ is the arc-length parameterization of the interface $\Gamma$, and $Q(\mathbf{X}(s))$ is the source strength on the interface. The expression above can also be written as

$$
f=f_{c}-Q \delta_{\Gamma}
$$

\footnotetext{
* Center For Research in Scientific Computation \& Department of Mathematics, North Carolina State University, Raleigh, NC 27695-8205, USA, (zhilin@math.ncsu.edu).

${ }^{\dagger}$ Department of Mathematics, Virginia Polytechnic Institute and State University, Blacksburg, VA, USA, (tlin@math.vt.edu).

$¥$ Applied Mathematics, Caltech, Pasadena, CA 91125. Current address: Exxon Production Research Company, Houston, TX., USA.
} 
Such a source function is one of the most important features of Peskin's immersed boundary method (IBM) [40, 41], which has been used for many problems in mathematical biology and computational fluid mechanics, see for example, $[5,13,14,16,43]$ and many others.

When $\beta \in C^{2}$ in $\Omega^{-} \cup \Omega^{+}$excluding the interface $\Gamma$, see Fig.1.1, then $u(x, y) \in H^{1}$, see [6]. From equation (1.1) and (1.2), it is easy to obtained the jump conditions

$$
\begin{aligned}
{[u]_{\Gamma} } & =u(x, y)^{+}-u(x, y)^{-}=0, \quad \text { continuity condition, } \\
{\left[\beta \frac{\partial u}{\partial \mathbf{n}}\right]_{\Gamma} } & =\beta^{+} \frac{\partial u^{+}}{\partial \mathbf{n}}-\beta^{-} \frac{\partial u^{-}}{\partial \mathbf{n}}=Q(s), \quad \text { net flux across the interface, }
\end{aligned}
$$

where the jump is defined as the difference of the limiting values from the outside of the interface to the inside, and $\frac{\partial u^{+}}{\partial \mathbf{n}}$ is the normal derivative of the solution. Therefore, the model interface problem can be written in an equivalent form:

$$
\begin{gathered}
-\nabla \cdot(\beta \nabla u)=f_{c}, \quad(x, y) \in \Omega-\Gamma, \quad f_{c} \in L^{2}(\Omega), \\
{[u]_{\Gamma}=0, \quad\left[\beta \frac{\partial u}{\partial \mathbf{n}}\right]_{\Gamma}=Q(s),\left.\quad u\right|_{\partial \Omega}=0 .}
\end{gathered}
$$

In this paper, we will use the two known jump conditions (1.4)-(1.5) to develop new finite element methods to solve the interface problem above.

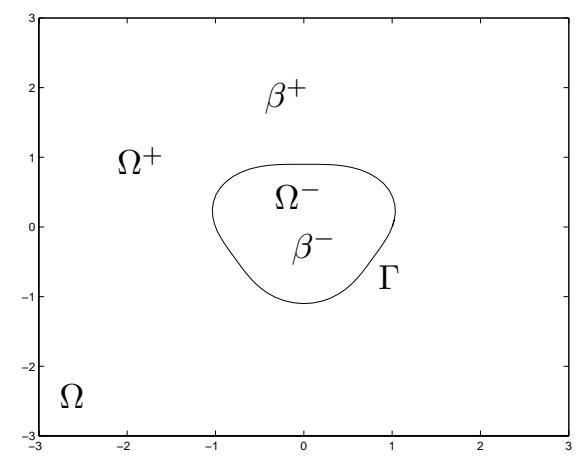

FIG. 1.1. A rectangular domain $\Omega=\Omega^{+} \cup \Omega^{-}$with an immersed interface $\Gamma$. The coefficients $\beta(\mathbf{x})$ may have a jump across the interface.

A related problem is the parabolic equation

$$
\mathbf{u}_{t}+\mathbf{c} \cdot \nabla \mathbf{u}=\nabla \cdot(\beta \nabla \mathbf{u})+\mathbf{f}(\mathbf{x}, t)
$$

in which the interface may be fixed or moving with time $t$, and the coefficient $\mathbf{c}$ and $\beta$ may have different values across the interface. An efficient discretization for (1.1) is essential to the numerical solution of this parabolic type equation.

Solving the Poisson equation (1.1) with discontinuous coefficients and/or singular source terms, usually is not only the slowest part of the entire simulation for many applications, but also leads to the loss in accuracy. Solving interface problems efficiently and accurately is still a challenge because of many irregularities associated with them. Many numerical methods have been developed, and below is a brief review on those closely related to this paper.

1.1. Body fitting grid methods based on finite element discretization. It is well known that a second order accurate approximation to the solution of an interface problem can be generated by 
the Galerkin finite element method with the standard linear basis functions if the triangulation is aligned with the interface (body fitting grid), $[2,6,7,18,47]$. One advantage of the finite element formulation is that the resulting linear system of equation is symmetric positive definite for a self-adjoint elliptic operator which ensures the stability of the algorithm. Applications of such methods can be found in [38] and many others.

However, it is difficult and time consuming to generate a body fitting grid for an interface problem in which the interface separates the solution domain into pieces or problems with complicated geometries. Such a difficulty becomes even more severe for moving interface problems because a new grid has to be generated at each time step. Few publications can be found on using body fitting grids to solve moving interface problems with topological changes such as merging and splitting.

Domain decomposition methods can also be used for solving interface problems, for example, [11]. On each sub-domain, one needs to solve the differential equation defined on an irregular domain. The information then is transfered between the different domains.

1.2. Cartesian grid methods based on finite difference discretization. Using Cartesian or adaptive Cartesian grids for interface problems has the following merits:

- There is almost no cost in the grid generation. This is very significant for moving boundary/interface problems.

- There are many efficient and popular packages/solvers and numerical methods which are written for Cartesian grids, for examples, fast Poisson solvers such as fishpack, Navier-Stokes equation solvers in two and three dimensions on a rectangular square or a box, Clawpack [24] for conservation laws, and FFT packages etc. It is relatively easier to incorporate new methods using existing packages/solvers based on the same grid.

- Recently, the level set method, first proposed in [39], has been successfully used to treat a number of moving interface/boundary problems, especially for problems with topological changes, and for problems in three dimensions. The level set method works best with Cartesian grids.

- It is easier to generate super convergent approximations to important physical quantities such as fluxes using Cartesian grids.

It is true that numerical methods based on Cartesian grids may have some difficulties to adjust and may lose accuracy for curved interfaces/boundaries. Due to non-smoothness of the solutions, many standard finite difference algorithms and analysis do not apply for interface problems. A lot of efforts have been made in this regard for various problems. Below we just review some methods in the literature which are related to this paper.

The smoothing method for discontinuous coefficients. A simple approach is to smooth out the coefficient, see for example [44]. The level set expression of interfaces makes the smoothing method much easier for two and three dimensional problems. However, solutions are also smeared out by the smoothing method. Another commonly used and sophisticated method is the harmonic averaging technique [3, 42]. While this method is second order accurate for certain one dimensional problems, usually it is not for two and three dimensional problems.

Peskin's immersed boundary method. If there is only a singular source term and the coefficient is continuous, a very simple and first order method is Peskin's immersed boundary method (IBM) using a discrete delta function $[40,41]$.

Fast solvers based on boundary integral equations. Based on integral equations, some fast solvers are available for Poisson equations with piecewise constant coefficients and other problems $[17,35$, 
$36,37]$. In these methods, an integral equation is set up at some points on the interface and the boundary for unknown source strength, and the solution then can be found using a fast boundary integral technique. Non-homogeneous source terms can be decomposed as two homogeneous problems.

Immersed interface methods. The original motivation of the immersed interface method (IIM) $[25,28]$ is to develop a second order finite difference scheme for very general second order elliptic and parabolic linear PDEs in which an integral equation may not be available, for example, (1.1) with variable coefficient $\beta$. In this regard, the method is successful and has been applied to problems ranging from one, two, and three dimensional problems [29]; elliptic, parabolic [46], hyperbolic [27], and mixed type equations [34]; fixed and moving interfaces [30], and many applications [19, 26, 32, 33]. However, with variable piecewise coefficients, the resulting linear system of equation from IIM is not symmetric positive definite. While it is stable for one dimensional problems and certain problems in two dimensions [22], the stability of the algorithm may depend on the choice of one or more additional grid points in addition to the standard finite difference scheme [15]. In this regard, the method is not very robust. Various attempts, such as the multigrid method by L. Adams [1] and the explicit jump immersed interface method (EJIIM) [45], to mention just a few, have been made to improve the stability and to speed up the method.

It is the purpose of this paper to combine the advantages of simple structure of Cartesian grids and the finite element formulation to develop accurate, stable numerical methods for interface problems. More precisely, we want to develop new methods that are accurate at all grid points including those near or on the interface; stable with nice algebraic structure (the resulting linear system of equation is symmetric positive definite for self-adjoint elliptic equations) even with discontinuous coefficients. We also hope the methods developed here can be built into other Cartesian grids based methods such as LeVeque's Clawpack and Berger's AMR (adaptive mesh refinement) and other packages. The error analysis presented in this paper requires the interface $\Gamma$ to be smooth; the solution to be piecewisely twice differentiable ${ }^{1}$. The algorithms proposed in this paper, however, do not have any particular restrictions compared with standard finite element methods.

We now take a look at the weak formulation of the interface problem. Assuming $f(x, y)$ in (1.1) has the form of (1.2), we multiply a test function $v(x, y) \in H_{0}^{1}(\Omega)$ to both sides of the first equation in (1.6) and integrate over the domain $\Omega^{+}$and $\Omega^{-}$, respectively. Since $f_{c} \in L^{2}(\Omega)$, we have

$$
\iint_{\Omega} f_{c} v d x d y=\iint_{\Omega^{+}} f_{c} v d x d y+\iint_{\Omega^{-}} f_{c} v d x d y
$$

Applying the Green's theorem in the domain $\Omega^{+}$, the outside of the closed interface $\Gamma$, we get

$$
-\int_{\partial \Omega} \beta v \frac{\partial u}{\partial \mathbf{n}} d s-\int_{\Gamma} \beta^{+} v^{+} \frac{\partial u^{+}}{\partial \mathbf{n}^{+}} d s+\iint_{\Omega^{+}} \beta \nabla u \cdot \nabla v d x d y=\iint_{\Omega^{+}} f_{c} v d x d y,
$$

where $\mathbf{n}^{+}$and $\mathbf{n}^{-}=\mathbf{n}$ are the unit normal directions of the interface $\Gamma$ pointing inward and outward respectively. Similarly there is the following relation from the inside of the interface $\Omega^{-}$:

$$
-\int_{\Gamma} \beta^{-} v^{-} \frac{\partial u^{-}}{\partial \mathbf{n}^{-}} d s+\iint_{\Omega^{-}} \beta \nabla u \cdot \nabla v d x d y=\iint_{\Omega^{-}} f_{c} v d x d y .
$$

Since

$$
-\int_{\Gamma} \beta^{+} v^{+} \frac{\partial u^{+}}{\partial \mathbf{n}^{+}} d s=\int_{\Gamma} \beta^{+} v^{+} \frac{\partial u^{+}}{\partial \mathbf{n}^{-}} d s=\int_{\Gamma} \beta^{+} v^{+} \frac{\partial u^{+}}{\partial \mathbf{n}} d s,
$$

\footnotetext{
${ }^{1}$ Such a solution is usually in $H^{1}$ but not in $H^{2}$.
} 
by applying the zero boundary condition $\left.v\right|_{\partial \Omega}=0$ and adding the (1.9) and (1.10) together, we get:

$$
\int_{\Gamma} \beta^{+} v^{+} \frac{\partial u^{+}}{\partial \mathbf{n}} d s-\int_{\Gamma} \beta^{-} v^{-} \frac{\partial u^{-}}{\partial \mathbf{n}} d s+\iint_{\Omega} \beta \nabla u \cdot \nabla v d x d y=\iint_{\Omega} f_{c} v d x d y .
$$

Using the flux jump condition (1.5), we obtain the weak form for the interface problem

$$
\iint_{\Omega} \beta(x, y) \nabla u \cdot \nabla v d x d y=\iint_{\Omega} f_{c} v d x d y-\int_{\Gamma} v Q d s, \quad \forall v(x) \in H_{0}^{1}(\Omega) .
$$

The weak form does allow discontinuities in the coefficient and the derivatives of the solution. The existence of the weak solution is discussed in $[6,7]$. Theoretically, the weak form is the same as those discussed in many standard finite element method text books, see $[6,7,23]$ for example, and will be used to derive related finite element methods later in this paper.

This paper is organized as follows. In Sec. 2, a non-conforming finite element space is introduced. The related basis functions satisfy the homogeneous jump conditions (1.4)-(1.5) with $Q(s) \equiv 0$ either exactly or approximately. The interpolation function in the new finite element space is constructed and analyzed. A non-trivial numerical example using the Galerkin finite element method is also given. Similar discussions are carried out for a conforming finite element space in Sec. 3. The key idea is to extend the support of the basis function if necessary. The Galerkin finite element method has been shown to be convergent in the $H^{1}$ norm for certain interface problems. Our discussion leads to another finite element method that uses Cartesian triangulation away from the interface, but introduces a few additional nodal points near the interface according to certain rules. The standard linear basis functions then can be defined on the new triangulation. The comparison of the two conforming finite elements are discussed and supported by a numerical example.

It is worthwhile to point out that although the discussions in this paper are based on Cartesian grids, the methods and the analysis can be easily extended to other grids that are not necessarily aligned with the interfaces.

2. A non-conforming immersed finite element space and analysis. In this section, we introduce a finite element space whose basis are piecewise linear functions satisfying the homogeneous jump conditions either exactly or approximately. Without loss of generality, we assume that the domain $\Omega$ is a rectangle which is separated by an interface $\Gamma$ into two sub-domains $\Omega^{+}$and $\Omega^{-}$such that $\Omega=\Omega^{+} \cup \Omega^{-} \cup \Gamma$, see Fig. 1.1. Also, without loss of generality, we assume that $Q=0$, and the coefficient $\beta$ in the boundary value problem (1.6) has two pieces separated by the interface $\Gamma$

$$
\beta(\mathbf{x})= \begin{cases}\beta^{+}, & \text {if } \mathbf{x} \in \Omega^{+}, \\ \beta^{-}, & \text {if } \mathbf{x} \in \Omega^{-},\end{cases}
$$

with $\beta(\mathbf{x}) \geq \beta_{0}>0$ for any $\mathbf{x} \in \mathbf{\Omega}$.

A Cartesian grid is then used to form a uniform triangular partition $\mathcal{T}_{h}$ with step size $h$ on $\Omega$ such that each element $T \in \mathcal{T}_{h}$ is a triangle constructed by the two legs and one of the diagonals in a subrectangle. The discussions and results of this paper can obviously be extended to other grids that are not necessarily aligned with the interface.

We call an element $T \in \mathcal{T}_{h}$ an interface element if the interface $\Gamma$ passes through the interior of $T$, see Fig. 2.1 for a typical geometric configuration; otherwise we call $T$ a non-interface element. We assume that the interface meets the edges of an interface element at no more than two intersections ${ }^{2}$. Such an

\footnotetext{
${ }^{2}$ If one of edges is part of the interface, then the element is a non-interface element.
} 
assumption is reasonable if $h$ is small and guaranteed if the interface is expressed in terms of the zero level set of the signed distance function (or approximately) of the interface. As in the common practice, we approximate the interface in $T$ by a line segment connecting the intersections of the interface and the edges of the triangles, for example, the line segment $\overline{D E}$ in Fig. 2.1. The line segment divides $T$ into two parts $T^{+}$and $T^{-}$with $T=T^{+} \cup T^{-} \cup \overline{D E}$. There is a small region in $T$

$$
T_{r}=T-\Omega^{+} \cap T^{+}-\Omega^{-} \cap T^{-}
$$

whose area is of order $O\left(h^{3}\right)$. This indicates that the interface is perturbed in a magnitude of $O\left(h^{2}\right)$. From [7] and the discussions later in this section, such a perturbation will only affect the solution, and the interpolation function to an order of $h^{2}$. In this paper, $T, T^{+}, T^{-}$, and $T_{r}$ are all defined as closed sets.

As usual, we want to construct local basis functions on each element $T$ of the partition $\mathcal{T}_{h}$. For a non-interface element $T \in \mathcal{T}_{h}$, we simply use the standard linear shape functions on $T$, and use $S_{h}(T)$ to denote the linear spaces spanned by the three nodal basis functions on $T$. Attention is needed only for interface elements, and we will discuss it in the following sub-section.

2.1. Local basis functions on an interface element. We assume that $\beta$ is piecewise constant. Without loss of generality, we consider a reference interface element $T$ whose geometric configuration is given in Fig. 2.1 in which the curve between points $D$ and $E$ is a part of the interface. The basis function in a general interface element can then be defined through the usual affine transformation. We assume that the coordinates at $A, B, C, D$, and $E$ are

$$
(0, h), \quad(0,0), \quad(h, 0), \quad\left(0, y_{1}\right), \quad\left(h-y_{2}, y_{2}\right),
$$

with the restriction

$$
0 \leq y_{1} \leq h, \quad 0 \leq y_{2}<h
$$

Once the values at vertices $A, B$, and $C$ of the element $T$ are specified, we construct the following piecewise linear function:

$$
\begin{aligned}
& u(\mathbf{x})= \begin{cases}u^{+}(\mathbf{x})=a_{0}+a_{1} x+a_{2}(y-h), & \text { if } \mathbf{x}=(x, y) \in T^{+}, \\
u^{-}(\mathbf{x})=b_{0}+b_{1} x+b_{2} y, & \text { if } \mathbf{x}=(x, y) \in T^{-},\end{cases} \\
& u^{+}(D)=u^{-}(D), \quad u^{+}(E)=u^{-}(E), \quad \beta^{+} \frac{\partial u^{+}}{\partial \mathbf{n}}=\beta^{-} \frac{\partial u}{\partial \mathbf{n}},
\end{aligned}
$$

where $\mathbf{n}$ is the unit normal direction of the line segment $\overline{D E}$. This is a piecewise linear function in $T$ that satisfies the homogeneous jump conditions along $\overline{D E}$. Intuitively, there are six constraints and six parameters, so we can expect the solution exists and is unique as confirmed in the following theorem.

TheOREM 2.1. Given a right triangle $A B C$ as indicated in Fig. 2.1. The piecewise linear function $u(x, y)$ defined by (2.4) and (2.5) is uniquely determined by $u(A), u(B)$ and $u(C)$.

Proof: Let $\mathbf{x}=(x, y)^{T}$. Because $u^{+}$and $u^{-}$are linear functions, we have

$$
u(\mathbf{x})= \begin{cases}u^{+}(x, y)=u(A)+a_{1} x+a_{2}(y-h), & \mathbf{x} \in T^{+}, \\ u^{-}(x, y)=u(B)+\frac{u(C)-u(B)}{h} x+b_{2} y, & \mathbf{x} \in T^{-} .\end{cases}
$$

From the continuity condition at $D$ and $E$, we have two equations

$$
\begin{aligned}
a_{2}\left(y_{1}-h\right)-b_{2} y_{1} & =u(B)-u(A), \\
a_{1}\left(h-y_{2}\right)+a_{2}\left(y_{2}-h\right)-b_{2} y_{2} & =u(B)-u(A)+b_{1}\left(h-y_{2}\right),
\end{aligned}
$$




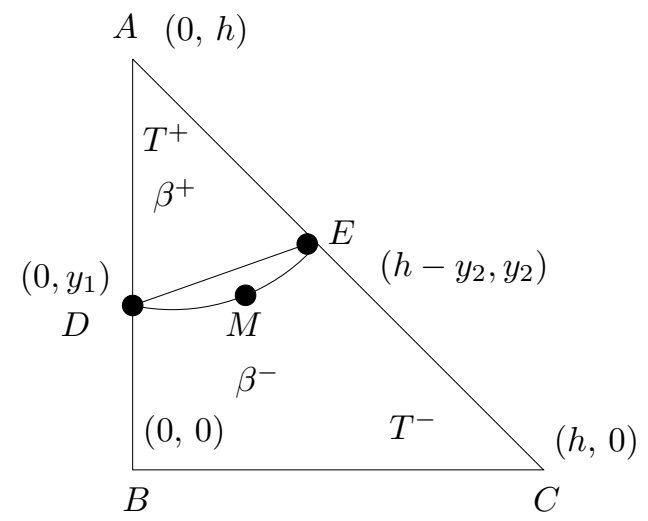

FIG. 2.1. A typical triangle element with an interface cutting through. The curve between $D$ and $E$ is part of the interface curve $\Gamma$ which is approximated by the line segment $\overline{D E}$. In this picture, $T$ is the triangle $\triangle A B C, T^{+}=\triangle A D E$, $T^{-}=T-T^{+}$, and $T_{r}$ is the region enclosed by the $\overline{D E}$ and the arc DME.

where

$$
b_{1}=\frac{u(C)-u(B)}{h} .
$$

The third equation is from the flux jump condition:

$$
a_{1} \alpha-a_{2}+\rho b_{2}=\rho \alpha b_{1},
$$

where $\rho=\beta^{-} / \beta^{+}$, and we have used the fact that the normal direction of the line segment is $(\alpha,-1)$ with $\alpha=\left(y_{2}-y_{1}\right) /\left(h-y_{2}\right)$. The coefficient matrix of the linear system for the unknowns $a_{1}, a_{2}$, and $b_{2}$ is

$$
\mathcal{A}=\left[\begin{array}{ccc}
0 & y_{1}-h & -y_{1} \\
h-y_{2} & y_{2}-h & -y_{2} \\
\alpha & -1 & \rho
\end{array}\right] .
$$

Evaluating the determinant of the matrix above, using the relation of $h-y_{1}=\left(h-y_{2}\right)(1+\alpha)$, we obtain the following after some manipulations

$$
\begin{aligned}
\operatorname{det}(\mathcal{A})= & -\left(y_{1}-h\right) y_{2} \alpha+\left(h-y_{2}\right) y_{1}+\alpha\left(y_{2}-h\right) y_{1}-\rho\left(h-y_{2}\right)\left(y_{1}-h\right) \\
= & \left(h-y_{2}\right) y_{2} \alpha(1+\alpha)+\left(h-y_{2}\right) y_{1}-\alpha\left(h-y_{2}\right)\left(y_{2}-\alpha\left(h-y_{2}\right)\right) \\
& +\rho\left(h-y_{2}\right)^{2}(1+\alpha) \\
= & \left(h-y_{2}\right)\left(y_{1}+h \alpha^{2}+\rho\left(h-y_{2}\right)(1+\alpha)\right)>0 .
\end{aligned}
$$

Thus from the theory of linear algebra, there is a unique solution to the linear system (2.7), (2.8) and (2.10).

We now introduce a local finite element space on each element $T$ of the partition $T_{h}$ as follows:

$$
S_{h}(T)= \begin{cases}\{u(\mathbf{x}) \mid u(\mathbf{x}) \text { is linear on } T\}, & \text { if } T \text { is a non-interface element } \\ \{u(\mathbf{x}) \mid u(\mathbf{x}) \text { is defined by }(2.4)-(2.5)\}, & \text { if } T \text { is an interface element. }\end{cases}
$$

It is well known that the dimension of $S_{h}(T)$ is three if $T$ is a non-interface element. When $T$ is an interface element, $S_{h}(T)$ contains three basis functions whose value at one of the vertices of $T$ is unity, and zero at the other two vertices. Furthermore, Theorem 2.1 tells us that any function in $S_{h}(T)$ is a linear combination of these three basis functions. Therefore the dimension of $S_{h}(T)$ is also three even if $T$ is an interface element. 
2.2. The non-conforming finite element space. To describe the finite element space on the whole domain $\Omega$, we let $\Omega^{\prime}$ be the union of all the interface elements. Then we define the immersed finite element space $S_{h}(\Omega)$ as a set of functions such that

$$
S_{h}(\Omega)=\left\{\phi(\mathbf{x}) \quad\left|\forall T \in \mathcal{T}_{h}, \quad \phi\right|_{T} \in S_{h}(T),\left.\phi\right|_{\Omega \backslash \Omega^{\prime}} \in H^{1}\left(\Omega \backslash \Omega^{\prime}\right)\right\} .
$$

It is worthwhile to point out again that this finite element space is formed by piecewise linear functions defined according to the partition $\mathcal{T}_{h}$ and the interface, but the partition does not have to align along with the interface. Part of the interface can be immersed in some elements of $\mathcal{T}_{h}$, and this is the reason we call $S_{h}(\Omega)$ an immersed finite element (IFE) space. On the other hand, the IFE space is rather similar to the usual linear finite element $(\mathrm{FE})$ space defined by the partition $\mathcal{T}_{h}$. First, they are exactly the same on every non-interface element. Secondly, they have the same dimension. Fig 2.2 shows a typical basis function of $S_{h}(\Omega)$ with an interface cutting through its non-zero support region. Finally, if $\beta(\mathbf{x})$ has no discontinuity, then the IFE space becomes the usual linear finite element space. However, for a discontinuous $\beta(\mathbf{x})$, the IFE space is more sophisticated than the usual FE space since the jump conditions across the interface are satisfied to certain extent. In this case, the IFE space is similar to a non-conforming FE space in the way that the basis functions may not be continuous across the edges of elements in $\mathcal{T}_{h}$. Hence the IFE space introduced here is generally a non-conforming FE space.

The dimension of the non-conforming IFE space is the number of interior points for the Dirichlet problem. The basis function centered at a node is defined as:

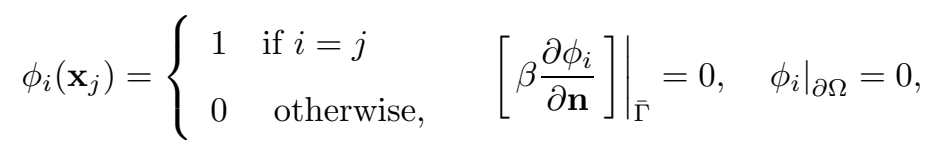

and $\phi_{i}$ is continuous in each element $T$ except some edges if $\mathbf{x}_{i}$ is a vertex of one or several interface triangles, see Fig. 2.2. We use $\bar{\Gamma}$ to denote the union of the line segment that is used to approximate the interface.

(a)

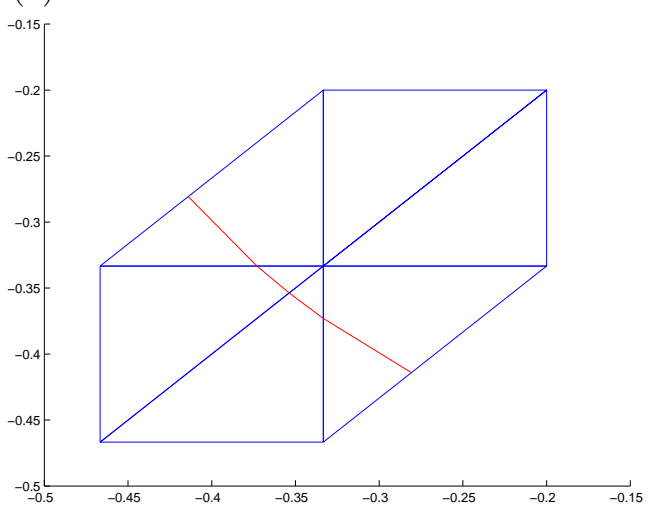

(b)

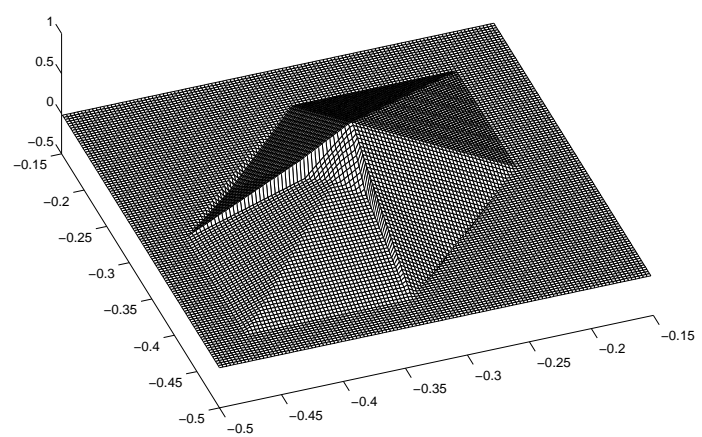

FIG. 2.2. (a) : A standard domain of six triangles with an interface cutting through. $(b):$ A global basis function on its support in the non-conforming immersed finite element space. The basis function has small jump across some edges.

2.3. Approximation capability of the non-conforming IFE space. Given a function $u(\mathbf{x})$ which is continuous on the entire domain and satisfies the flux jump condition, we define its interpolant in the IFE space $S_{h}(\Omega)$ as the function $u_{I}(\mathbf{x}) \in S_{h}(\Omega)$ such that

$$
u_{I}(\mathbf{x})=u(\mathbf{x}), \quad \text { if } \mathbf{x} \text { is a node of } \mathcal{T}_{h} .
$$


We would like to know how well $u_{I}(\mathbf{x})$ can approximate $u(\mathbf{x})$. Since $u_{I}(\mathbf{x})$ is the usual linear function on each non-interface element, we have the following standard error estimate [9]

$$
\left\|u_{I}-u\right\|_{0, T}+h\left\|I_{h} u-u\right\|_{1, T} \leq C_{1} h^{2}\|u\|_{2, T},
$$

where $\|\cdot\|_{s, A}$ is the norm of the Sobolev space $H^{s}(A)$ defined in a set $A$, and $C_{1}$ is a constant ${ }^{3}$. Similar error estimates can also be given in the norm of the space $W^{s, p}(A)$. When $T$ is an interface element, each partial derivative of $u_{I}$ on $T$ is a piecewise constant function consisting of two values, $\partial u_{I}^{+} / \partial x$ and $\partial u_{I}^{-} / \partial x$, or $\partial u_{I}^{+} / \partial y$ and $\partial u_{I}^{-} / \partial y$, where $u_{I}^{i}$ are the restrictions of $u_{I}$ on $T^{i}, i=+,-$. The following theorem provides the error estimates on them.

THEOREM 2.2. Let $T \in \mathcal{T}_{h}$ be an interface element, and let $u(x, y)$ be a continuous function such that its restriction $u^{i}=\left.u\right|_{T^{i}}$ on $T^{i}, \quad i=+,-$ are twice differentiable in each sub-domain $\Omega^{+} \cap T$ and $\Omega^{-} \cap T$, and satisfies the homogeneous jump conditions (1.4)-(1.5). Then we have the following error estimates

$$
\begin{aligned}
& \left\|\frac{\partial u_{I}}{\partial x}-\frac{\partial u}{\partial x}\right\|_{\infty, T \backslash T_{r}} \leq\left(22 \rho_{\text {cond }}^{2}+1\right)\left\|\mathcal{D}^{2} u\right\|_{\infty, T} h, \\
& \left\|\frac{\partial u_{I}}{\partial y}-\frac{\partial u}{\partial y}\right\|_{\infty, T \backslash T_{r}} \leq\left(22 \rho_{\text {cond }}^{2}+1\right)\left\|\mathcal{D}^{2} u\right\|_{\infty, T} h,
\end{aligned}
$$

where

$$
\rho_{\text {cond }}=\frac{\rho_{\max }}{\rho_{\min }}, \quad \rho_{\max }=\max _{\mathbf{x} \in \Omega}\left\{\rho, \frac{1}{\rho}\right\}, \quad \rho_{\min }=\min _{\mathbf{x} \in \Omega}\left\{\rho, \frac{1}{\rho}\right\} .
$$

and

$$
\left\|\mathcal{D}^{2} u\right\|_{\infty, T}=\max _{i=+,-}\left\{\left\|u_{x x}^{i}\right\|_{\infty, T \cap \Omega^{i}}+2\left\|u_{x y}^{i}\right\|_{\infty, T \cap \Omega^{i}}+\left\|u_{y y}^{i}\right\|_{\infty, T \cap \Omega^{i}}\right\}
$$

Proof: Again, we assume that the interface element $T$ has the configuration given in Fig. 2.1. Since $u(x, y)$ is twice differentiable in $\Omega^{+}$and $\Omega^{-}$, it is also twice differentiable in $T^{i} \backslash T_{r}$. First we choose a point $M$ on the interface such that the tangential line of the interface at $M$ is parallel to the line segment $\overline{D E}$ whose slope is:

$$
\alpha=\frac{y_{2}-y_{1}}{h-y_{2}}, \quad-1<\alpha<+\infty
$$

Notice that $y_{2}=\left(y_{1}+\alpha h\right) /(1+\alpha)$. Plugging this into $(2.12)$ and re-arranging terms we get

$$
\begin{aligned}
|\operatorname{det}(\mathcal{A})| & =\left(h-y_{2}\right)\left(y_{1}+h \alpha^{2}+\rho\left(h-\frac{y_{1}+\alpha h}{1+\alpha}\right)(1+\alpha)\right) \\
& =\left(h-y_{2}\right)\left(y_{1}+h \alpha^{2}+\rho\left(h-y_{1}\right)\right) \\
& \geq \rho_{\min }\left(h-y_{2}\right)\left(h \alpha^{2}+h\right),
\end{aligned}
$$

where $\mathcal{A}$ is the matrix given in (2.11).

Now consider the following Taylor expansions of $u(x, y)$ at $A$ and $B$

$$
\begin{aligned}
& u^{+}(x, y)=u(A)+\frac{\partial u^{+}(A)}{\partial x} x+\frac{\partial u^{+}(A)}{\partial y}(y-h)+\ldots, \\
& u^{-}(x, y)=u(B)+\frac{\partial u^{-}(B)}{\partial x} x+\frac{\partial u^{-}(B)}{\partial y} y+\ldots
\end{aligned}
$$

\footnotetext{
${ }^{3}$ We use $C_{1}$ instead of $C$ because we have already used $C$ in Fig. 2.1.
} 
At point $D, u^{+}(D)=u^{-}(D)$, so we can write

$$
\frac{\partial u^{+}(A)}{\partial y}\left(y_{1}-h\right)-\frac{\partial u^{-}(B)}{\partial y} y_{1}=u(B)-u(A)+e_{1},
$$

where $e_{1}$ is the error terms from the two Taylor expansions, therefore $\left|e_{1}\right| \leq 2\left\|\mathcal{D}^{2} u\right\|_{\infty, T} h^{2}$. Similarly at point $E$, we have the relation

$$
\begin{gathered}
\frac{\partial u^{+}(A)}{\partial x}\left(h-y_{2}\right)+\frac{\partial u^{+}(A)}{\partial y}\left(y_{2}-h\right)-\frac{\partial u^{-}(B)}{\partial y} y_{2}= \\
u(B)-u(A)+\frac{\partial u^{-}(B)}{\partial x}\left(h-y_{2}\right)+\tilde{e_{2}},
\end{gathered}
$$

where $\tilde{e_{2}}$ is the error terms from the two Taylor expansions. Notice that

$$
b_{1}=\frac{u(C)-u(B)}{h}=\frac{\partial u^{-}(B)}{\partial x}+\frac{\partial^{2} u^{-}(R)}{\partial x^{2}} h,
$$

where $R$ is some point between $B$ and $C$. Therefore we can write

$$
\begin{gathered}
\frac{\partial u^{+}(A)}{\partial x}\left(h-y_{2}\right)+\frac{\partial u^{+}(A)}{\partial y}\left(y_{2}-h\right)-\frac{\partial u^{-}(B)}{\partial y} y_{2}= \\
u(B)-u(A)+b_{1}\left(h-y_{2}\right)+e_{2},
\end{gathered}
$$

where $e_{2} \leq 2\left\|\mathcal{D}^{2} u\right\|_{\infty, T} h^{2}$ after the term $\partial^{2} u^{-}(R) / \partial x^{2}$ is absorbed into other second order derivative terms. Using (2.20) and (2.21), and from the flux jump relation of $u$ at $M$, we have the third equation

$$
\begin{aligned}
\frac{\partial u^{+}(A)}{\partial x} \alpha-\frac{\partial u^{+}(A)}{\partial y}+\rho \frac{\partial u^{-}(B)}{\partial y} & =\rho \alpha \frac{\partial u^{-}(B)}{\partial x}+\tilde{e_{3}} \\
& =\rho \alpha b_{1}+e_{3},
\end{aligned}
$$

where $e_{3}$ is the accumulation of errors from the Taylor expansions. Hence,

$$
\left|e_{3}\right| \leq\left(\max \{|\alpha|, \quad 1\}+\rho_{\max }(|\alpha|+1)\right)\left\|\mathcal{D}^{2} u\right\|_{\infty, T} h .
$$

Equations (2.22), (2.25), and (2.26) are the same as those in (2.7), (2.8) and (2.10) with perturbation to the right hand sides. By subtracting (2.7), (2.8), and (2.10), from (2.22), (2.25), and (2.26) respectively, we get a linear system of equations for the errors of the first order of partial derivatives. The solution of the error $\partial u^{-}(B) / \partial y-\partial u_{I}^{-} / \partial y$ is

$$
\begin{aligned}
\frac{\partial u^{-}(B)}{\partial y}-\frac{\partial u_{I}^{-}}{\partial y} & =\left|\begin{array}{ccc}
0 & y_{1}-h & e_{1} \\
h-y_{2} & y_{2}-h & e_{2} \\
\alpha & -1 & e_{3}
\end{array}\right| / \operatorname{det}(A) \\
& =\frac{e_{2}\left(y_{1}-h\right) \alpha-e_{1}\left(h-y_{2}\right)-e_{1} \alpha\left(y_{2}-h\right)-e_{3}\left(h-y_{2}\right)\left(y_{1}-h\right)}{\operatorname{det}(A)} \\
& =\frac{-e_{2}\left(h-y_{2}\right)(1+\alpha) \alpha-e_{1}\left(h-y_{2}\right)+e_{1} \alpha\left(h-y_{2}\right)-e_{3}\left(h-y_{2}\right)^{2}(1+\alpha)}{\operatorname{det}(A)} \\
& =\frac{\left(h-y_{2}\right)\left[-\alpha(1+\alpha) e_{2}+(\alpha-1) e_{1}+\left(h-y_{2}\right)(1+\alpha) e_{3}\right]}{\operatorname{det}(\mathcal{A})},
\end{aligned}
$$

where we have used the relation $y_{1}=y_{2}-\alpha\left(h-y_{2}\right)$ again. Now it is easy to derive an upper bound of the error

$$
\left|\frac{\partial u^{-}(B)}{\partial y}-\frac{\partial u_{I}^{-}}{\partial y}\right| \leq \frac{\alpha^{2}+2|\alpha|+1+\frac{(1+|\alpha|)\left(\max \{|\alpha|, 1\}+\rho_{\max }(1+|\alpha|)\right.}{2}}{\rho_{\min }\left(\alpha^{2}+1\right)} 2\left\|\mathcal{D}^{2} u\right\|_{\infty, T} h .
$$


If $|\alpha| \leq 1$, then

$$
\begin{aligned}
\frac{\alpha^{2}+2|\alpha|+1+(1+|\alpha|)\left(\max \{|\alpha|, 1\}+\rho_{\max }(1+|\alpha|)\right) / 2}{\rho_{\min }\left(\alpha^{2}+1\right)} & \leq \rho_{\text {cond }} \frac{\frac{3}{2} \alpha^{2}+\frac{7}{2}|\alpha|+2}{\alpha^{2}+1} \\
& \leq \rho_{\text {cond }} \frac{5 \alpha^{2}+5-\frac{7}{2} \alpha^{2}+\frac{7}{2}|\alpha|-3}{\alpha^{2}+1} \\
& \leq \rho_{\text {cond }} \frac{5 \alpha^{2}+5}{\alpha^{2}+1} \\
& =5 \rho_{\text {cond }} .
\end{aligned}
$$

Note that we have used the fact that $f(x)=-\frac{7}{2} x^{2}+\frac{7}{2} x-3<0$ when $0 \leq x \leq 1$ in the proof above.

If $|\alpha|>1$, then

$$
\begin{aligned}
\frac{\alpha^{2}+2|\alpha|+1+(1+|\alpha|)\left(\max \{|\alpha|, 1\}+\rho_{\max }(1+|\alpha|)\right) / 2}{\rho_{\min }\left(\alpha^{2}+1\right)} & \leq \rho_{\text {cond }} \frac{2 \alpha^{2}+\frac{7}{2}|\alpha|+\frac{3}{2}}{\alpha^{2}+1} \\
& \leq \rho_{\text {cond }} \frac{5 \alpha^{2}+5-3|\alpha|^{2}+\frac{7}{2}|\alpha|-\frac{7}{2}}{\alpha^{2}+1} \\
& \leq 5 \rho_{\text {cond }} .
\end{aligned}
$$

In the proof above, we have used the fact that $f(x)=-3 x^{2}+\frac{7}{2} x-\frac{7}{2} \leq 0$ when $x \geq 1$.

In either case, $|\alpha| \leq 1$ or $|\alpha|>1$, we have an upper bound, not optimal though, which is

$$
\left|\frac{\partial u^{-}(B)}{\partial y}-\frac{\partial u_{I}^{-}}{\partial y}\right| \leq 10 \rho_{\text {cond }}\left\|\mathcal{D}^{2} u\right\|_{\infty, T} h .
$$

We continue to proceed with the following derivation

$$
\begin{aligned}
\left|\frac{\partial u_{I}^{-}}{\partial y}-\frac{\partial u^{-}(M)}{\partial y}\right| & \leq\left|\frac{\partial u_{I}^{-}}{\partial y}-\frac{\partial u^{-}(B)}{\partial y}\right|+\left|\frac{\partial u^{-}(B)}{\partial y}-\frac{\partial u^{-}(M)}{\partial y}\right| \\
& \leq 10 \rho_{\text {cond }}\left\|\mathcal{D}^{2} u\right\|_{\infty, T} h+\left\|\mathcal{D}^{2} u\right\|_{\infty, T} h \\
& \leq 11 \rho_{\text {cond }}\left\|\mathcal{D}^{2} u\right\|_{\infty, T} h,
\end{aligned}
$$

where the derivative of $\partial u^{-}(M) / \partial y$ is the following limit

$$
\frac{\partial u^{-}(M)}{\partial y}=\lim _{\mathbf{x} \in \Omega^{-}, \mathbf{x} \rightarrow M} \frac{\partial u(\mathbf{x})}{\partial y},
$$

and

$$
\left|\frac{\partial u_{I}^{-}}{\partial x}-\frac{\partial u^{-}(M)}{\partial x}\right|=\left|\frac{u(C)-u(B)}{h}-\frac{\partial u^{-}(M)}{\partial x}\right| \leq\left\|\mathcal{D}^{2} u\right\|_{\infty, T} h
$$

which give estimates in (2.15) and (2.16) for the case when $i=-$.

However, applying the same approach to $\frac{\partial u^{+}(A)}{\partial x}-\frac{\partial u_{I}^{+}}{\partial x}$ and $\frac{\partial u^{+}(A)}{\partial y}-\frac{\partial u_{I}^{+}}{\partial y}$ fails to generate the desired conclusion for the case when $i=+$. We now use the interface relations to prove the error estimates. Since $u$ is continuous, the directional derivative along the tangential direction of the interface is continuous at M

$$
-\frac{\partial u^{+}(M)}{\partial x} n_{y}+\frac{\partial u^{+}(M)}{\partial y} n_{x}=-\frac{\partial u^{-}(M)}{\partial x} n_{y}+\frac{\partial u^{-}(M)}{\partial y} n_{x},
$$


where $\left(n_{x}, n_{y}\right)=(\alpha,-1) / \sqrt{\alpha^{2}+1}$ is the unit normal direction of the interface $\Gamma$ at $M$. The flux jump condition at $M$ produces another equation

$$
\frac{\partial u^{+}(M)}{\partial x} n_{x}+\frac{\partial u^{+}(M)}{\partial y} n_{y}=\rho\left(\frac{\partial u^{-}(M)}{\partial x} n_{x}+\frac{\partial u^{-}(M)}{\partial y} n_{y}\right) .
$$

Solving $\partial u^{+}(M) / \partial x$ and $\partial u^{+}(M) / \partial y$ from (2.31) and (2.32) in terms of $\partial u^{-}(M) / \partial x$ and $\partial u^{-}(M) / \partial y$, we get

$$
\left[\begin{array}{c}
\frac{\partial u^{+}(M)}{\partial x} \\
\frac{\partial u^{+}(M)}{\partial y}
\end{array}\right]=\left[\begin{array}{cc}
n_{y}^{2}+\rho n_{x}^{2} & -n_{y} n_{x}+\rho n_{y} n_{x} \\
-n_{y} n_{x}+\rho n_{y} n_{x} & n_{x}^{2}+\rho n_{y}^{2}
\end{array}\right]\left[\begin{array}{c}
\frac{\partial u^{-}(M)}{\partial x} \\
\frac{\partial u^{-}(M)}{\partial y}
\end{array}\right]
$$

Notice that the maximum norm of the matrix in the expression above is bounded by $2 \rho_{\max }$, see $(2.17)$. From the definition of $u_{I}(x, y)$, equations (2.31) and (2.32) also hold when the function $u$ is replaced by $u_{I}(x, y)$. Therefore we have

$$
\begin{aligned}
\left\|\left[\begin{array}{c}
\frac{\partial u_{I}^{+}}{\partial x}-\frac{\partial u^{+}(M)}{\partial x} \\
\frac{\partial u_{I}^{+}}{\partial y}-\frac{\partial u^{+}(M)}{\partial y}
\end{array}\right]\right\|_{\infty} & \leq 2 \rho_{\max }\left\|\left[\begin{array}{l}
\frac{\partial u_{I}^{-}}{\partial x}-\frac{\partial u^{-}(M)}{\partial x} \\
\frac{\partial u_{I}^{-}}{\partial y}-\frac{\partial u^{-}(M)}{\partial y}
\end{array}\right]\right\|_{\infty} \\
& \leq 22 \rho_{\text {cond }}^{2}\left\|\mathcal{D}^{2} u\right\|_{\infty, T} h,
\end{aligned}
$$

because of the error estimates established for $u_{I}^{-}$. Finally we use the following triangle inequality

$$
\begin{aligned}
\left\|\left[\begin{array}{l}
\frac{\partial u_{I}^{i}}{\partial x}-\frac{\partial u^{i}(\mathbf{x})}{\partial x} \\
\frac{\partial u_{I}^{i}}{\partial y}-\frac{\partial u^{i}(\mathbf{x})}{\partial y}
\end{array}\right]\right\|_{\infty, T^{i} \backslash T_{r}} & \leq\left\|\left[\begin{array}{c}
\frac{\partial u_{I}^{i}}{\partial x}-\frac{\partial u^{i}(M)}{\partial x} \\
\frac{\partial u_{I}^{i}}{\partial y}-\frac{\partial u^{i}(M)}{\partial y}
\end{array}\right]\right\|_{\infty}+\left\|\left[\begin{array}{c}
\frac{\partial u^{i}(M)}{\partial x}-\frac{\partial u^{i}(\mathbf{x})}{\partial x} \\
\frac{\partial u^{i}(M)}{\partial y}-\frac{\partial u^{i}(\mathbf{x})}{\partial y}
\end{array}\right]\right\|_{\infty, T^{i} \backslash T_{r}} \\
\leq & \left(22 \rho_{\text {cond }}^{2}+1\right)\left\|\mathcal{D}^{2} u\right\|_{\infty, T} h,
\end{aligned}
$$

from (2.29) and (2.30) if $i=-$, or from (2.34) if $i=+$, and from a Taylor expansion for the second term above. Thus the proof of the theorem is completed.

Furthermore, we can easily use the estimates in Theorem 2.2 and the Taylor expansion to generate an error estimate for $u_{I}$ itself given in the following theorem.

THeOREM 2.3. Let $T \in \mathcal{T}_{h}$ be an interface element, and let $u(x, y)$ be a continuous function such that its restriction $u^{i}=\left.u\right|_{T^{i}}$ on $T^{i}, \quad i=+,-$ are twice differentiable in each sub-domain $\Omega^{+} \cap T$ and $\Omega^{-} \cap T$, and satisfies the homogeneous jump conditions (1.4)-(1.5). Then we have the following inequality:

$$
\left|u(\mathbf{x})-u_{I}(\mathbf{x})\right| \leq \begin{cases}C_{1} \tilde{h} h, & \text { if } \mathbf{x} \in T \backslash T_{r}, \\ C_{2} h^{2}, & \text { if } \mathbf{x} \in T_{r},\end{cases}
$$

where $\tilde{h}$ is the shortest distance between $\mathbf{x}$ and the vertices of $T$ which are on the same side of the interface as $\mathbf{x}$, and

$$
C_{1} \leq\left(1+44 \rho_{\text {cond }}^{2}\right)\left\|\mathcal{D}^{2} u\right\|_{\infty, T}
$$

and $C_{2}$ is some constant. 
Proof: Without loss of generality, we still use Fig.2.1 to illustrate our proof and assume that the point $B$ is the vertex closest to $\mathbf{x}$. If $\mathbf{x} \in T^{-} \backslash T_{r}$, then we have

$$
\begin{aligned}
u_{I}^{-}(\mathbf{x}) & =u_{I}(B)+{\frac{\partial u_{I}^{-}}{\partial x}}^{-}\left(x-x_{B}\right)+{\frac{\partial u_{I}^{-}}{\partial y}\left(y-y_{B}\right)}^{-} \\
& =u(B)+\frac{\partial u^{-}}{\partial x}(M)\left(x-x_{B}\right)+\frac{\partial u^{-}}{\partial y}(M)\left(y-y_{B}\right)+R_{1} \\
& =u(\mathbf{x})+R_{1}+R_{2},
\end{aligned}
$$

where $x_{B}, y_{B}$ are the coordinates of point $B$,

$$
\begin{aligned}
\left|R_{1}\right| & \leq 2 \max \left\{\left|\frac{\partial u_{I}^{-}}{\partial x}-\frac{\partial u^{-}(M)}{\partial x}\right|,\left|\frac{\partial u_{I}^{-}}{\partial y}-\frac{\partial u^{-}(M)}{\partial y}\right|\right\} h \\
& \leq 44 \rho_{\text {cond }}^{2}\left\|\mathcal{D}^{2} u\right\|_{\infty, T} \tilde{h} h,
\end{aligned}
$$

from (2.34), and

$$
\left|R_{2}\right| \leq\left\|\mathcal{D}^{2} u\right\|_{\infty, T} \tilde{h} h
$$

from the Taylor expansion. Similar result holds for $\mathbf{x} \in T^{+} \backslash T_{r}$.

If $\mathbf{x} \in T_{r}$, say $\mathbf{x} \in T \cap T^{-}$, but $\mathbf{x} \in \Omega^{+}$, for example. We take the closest point $R \in T$ to $\mathbf{x}$ from the line segment. We know that $\|\mathbf{x}-R\| \approx h^{2}$. Using the triangle inequality we obtain

$$
\begin{aligned}
\left|u_{I}(\mathbf{x})-u(\mathbf{x})\right| & \leq\left|u_{I}^{-}(\mathbf{x})-u_{I}^{-}(R)\right|+\left|u_{I}^{+}(R)-u(\mathbf{x})\right| \\
& \leq\left\|\mathcal{D}^{2} u\right\|_{\infty, T}\|\mathbf{x}-R\|+\left|u_{I}^{+}(R)-u^{+}(R)\right|+\left|u^{+}(R)-u^{+}(\mathbf{x})\right| \\
& \leq\left\|\mathcal{D}^{2} u\right\|_{\infty, T}\|\mathbf{x}-R\|+C_{1} \tilde{h} h+\left\|\mathcal{D}^{2} u\right\|_{\infty, T}\|\mathbf{x}-R\| \\
& \approx h^{2}
\end{aligned}
$$

In the proof, we have used the facts that $u_{I}^{+}(R)=u_{I}^{-}(R)$, the continuity condition for $u_{I}$ and $u(\mathbf{x})$, and the first error estimate of (2.35) of this theorem which has been already proved.

Notice that the intersections of the interface and the edges of the triangles are not in $T_{r}$ so they satisfy the first inequality in (2.35), a fact that we need to use in the next section.

REMARK 2.1. Although we have the error estimate for the interpolation functions for the nonconforming finite element method. The convergence analysis for the finite element solution is not straightforward for the particular non-conforming IFE space. Such error estimate is currently under investigation.

2.4. A non-conforming IFE method. It is obvious that the finite element space $S_{h}(\Omega)$ introduced in the last section is not in the space to which the solution of the interface problem belongs. A function $\phi$ of $S_{h}(\Omega)$ is continuous in the union of non-interface triangles but may be discontinuous on edges of interface triangles. Therefore the finite element method based on $S_{h}(\Omega)$ is non-conforming. For the interface problem, we now define its non-conforming IFE solution as a function $u_{h} \in S_{h 0}(\Omega)$ satisfying

$$
a_{h}\left(u_{h}, v_{h}\right)=\int_{\Omega} f v_{h} d x d y, \text { for all } v_{h} \in S_{h 0}(\Omega),
$$

where $S_{h 0}(\Omega)=\left\{\phi \in S_{h}(\Omega)|\phi|_{\partial \Omega}=0\right\}$, and

$$
a_{h}(u, v)=\sum_{T \in \mathcal{T}_{h}} \int_{T \in \mathcal{T}_{h}} \beta \nabla u \cdot \nabla v d x d y
$$


REMARK 2.2. If the flux jump is not homogeneous in (1.6), the non-conforming IFE solution $u_{h} \in$ $S_{h 0}(\Omega)$ then satisfies

$$
a_{h}\left(u_{h}, v_{h}\right)=\int_{\Omega} f v_{h} d x d y-\int_{\Gamma} v_{h} Q d s
$$

2.5. A numerical example for the non-conforming IFE method. We present a non-trivial example here to show the performance of the standard Galerkin finite element method using the nonconforming IFE space. In this example, we consider the boundary value problem defined by (1.1) with a Dirichlet boundary condition. The computational domain is the rectangle $-1 \leq x, y \leq 1$, and the interface is a circle centered at the origin with radius $r_{0}$. The boundary condition and the source term $f_{c}$ are determined from the exact solution

$$
u(x, y)= \begin{cases}\frac{r^{\alpha}}{\beta^{-}}, & \text {if } r \leq r_{0}, \\ \frac{r^{\alpha}}{\beta^{+}}+\left(\frac{1}{\beta^{-}}-\frac{1}{\beta^{+}}\right) r_{0}^{\alpha}, & \text { otherwise }\end{cases}
$$

where $r=\sqrt{x^{2}+y^{2}}$ and $\alpha=3$. Notice that the exact solution satisfies the homogeneous jump conditions (1.4)-(1.5).

The error estimates for the interpolation functions obtained in sub-section 2.3 indicate that the finite element solution in the IFE space may have a second order approximation capability. Hence we naturally expect the IFE solutions are second order accurate in the $L_{2}$ norm. Since the large errors occur near or at the interface which is one-dimensional lower than the solution domain, we only present the errors in the maximum norm in Fig. 2.3, in which the IFE solutions $u_{h}$ are found with various grid size $h$. The involved linear algebraic system has a structure similar to that in the Galerkin method with the usual linear finite element space. The jump in the coefficient of these tests is taken as $\rho=1: 1000$ or $\rho=1000: 1$, a quite large ratio. As discussed in [31], the errors in the numerical solutions generally do not decrease monotonously for interface problems. Therefore we need to use the linear regression or the least squares fitting to find the asymptotic convergence rate. In this way, we notice the second order convergence for one ratio, $\left\|u-u_{h}\right\|_{\infty} \sim h^{2}$, and super-linear convergence for the other, $\left\|u-u_{h}\right\|_{\infty} \sim h^{1.565}$, where $u$ is the exact solution of the boundary value problem. Similar behavior are observed for other ratios as well. The magnitude of the errors with 160 by 160 grid is about $10^{-4}$ for both ratios.

The non-conforming IFE method presented here is simple, easy to implement, and has an algebraic system similar to that of the Galerkin finite element method based on the standard finite element space. In particular, the partition of the IFE space does not have to be restricted by the geometry of the interface. The basis functions of the IFE space satisfy the jump conditions, which enables us to obtain sharp solutions near the interface. The same idea can be applied to treat three dimensional problems.

3. A conforming immersed finite element space and analysis. In this section, we develop a conforming IFE space to further improve accuracy of the finite element method based on the nonconforming IFE space described in the previous section. While the non-conforming IFE method performs better than the standard finite element method for interface problems, it does not seem to be second order in the infinite norm. Note that, for regular boundary value problems, the standard conforming finite element method using the piecewise linear has second order convergence in the infinite norm. We hope this is also true for the finite element method using a conforming IFE space with second order approximation capability. However, the requirements of the continuity and the jump relations (1.4)-(1.5) 
(a)

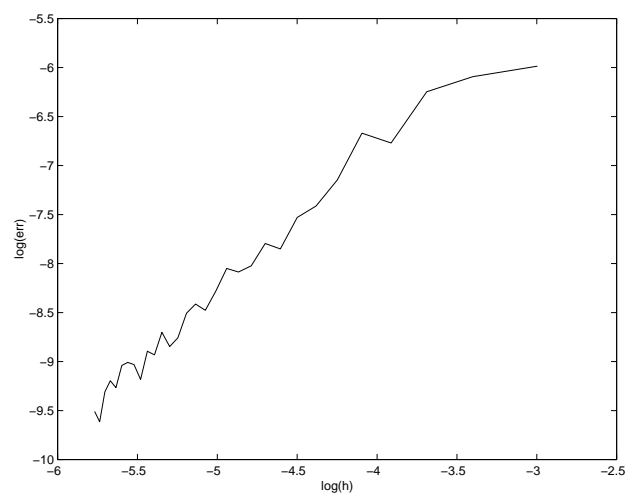

(b)

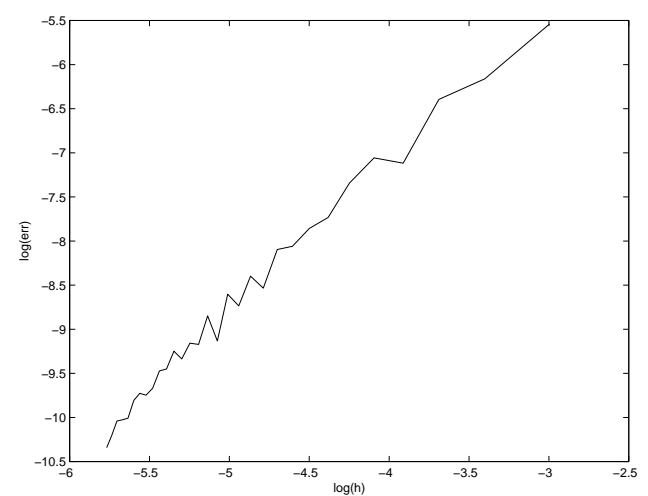

FIG. 2.3. The errors of the finite element solutions obtained in the non-conforming IFE space in the maximum norm versus the mesh size $h$ in $\log -\log$ scale with $r_{0}=\pi / 6.28, \alpha=3 . \quad(a): \beta^{-}=1, \beta^{+}=1000$. The linear regression analysis gives $\left\|u-u_{h}\right\|_{\infty} \approx 0.64657 h^{1.56459} ;(b): \beta^{-}=1000, \beta^{+}=1$. The linear regression analysis gives $\left\|u-u_{h}\right\|_{\infty} \approx$ $2.79434 h^{1.94833}$.

turn out to be rather difficult to satisfy simultaneously even with high order elements. One of the key ideas of our approach is to enlarge the support of some basis functions in the non-conforming finite element space so that the continuity condition can be maintained.

Let us examine the IFE space again to see why we need to enlarge the support of some basis functions. The non-conforming basis functions have the same compact support as the standard linear basis function. However, if we want the basis functions to have the same compactness, to be linear, and to satisfy

$$
\phi_{i}\left(\mathbf{x}_{j}\right)= \begin{cases}1, & \text { if } i=j, \\ 0, & \text { otherwise }\end{cases}
$$

in a conforming IFE space, then for a given function $u(x, y)$ that satisfies the jump conditions (1.4)-(1.5), we may not be able to construct a linear interpolation function that approximates $u(x, y)$ to second order in the maximum norm. To see this, let us consider an interface element $\triangle A B C$ as sketched in Fig. 3.1 (b) in which the line $\overline{D E}$ is part of the interface. Let $u(x, y)$ be a function satisfying the jump conditions

$$
[u]=0, \quad\left[\beta \frac{\partial u}{\partial \mathbf{n}}\right]=0,
$$

on the interface, and have the following values

$$
u(C)=h, \quad u(A)=u(B)=0 .
$$

It is very likely that $u(D) \sim h$ for an interface problem. The interpolation function has the form of

$$
u_{I}(x, y)=\sum u_{j} \phi_{j}(\mathbf{x})
$$

where $\phi_{j}$ are conforming basis functions. Then we must have $u_{I}(D)=\phi_{C}(D)=0$ since $u(A)=u(B)=0$ and all the basis functions that are not centered at $A$ and $B$ are zero on the entire line segment $\overline{A B}$. Hence $\left|u_{I}(D)-u(D)\right|=O(h)$ and the approximation is only first order accurate. Therefore, to achieve second order accuracy for the interpolation function, we need to either develop new non-conforming finite element methods, or extend the support of the basis functions. We will focus on the second approach and still use piecewise linear functions. High order elements shall be considered in the future.

Intuitively, it is not very difficulty to approximate any piecewise twice differentiable function to second order by piecewise polynomials. The challenge is how to maintain continuity along the edges and the 
jump conditions along the interface simultaneously. Our idea is to average the values of non-conforming basis functions with the same values at nodal points to keep the continuity. The details are described in the following subsections.

(a)

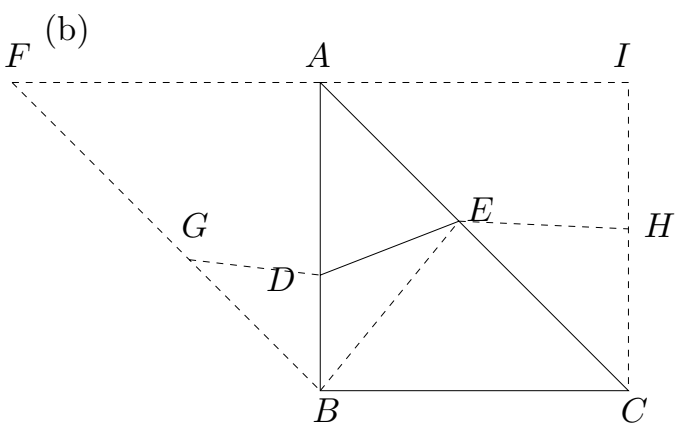

FIG. 3.1. (a) : An extended region of support of the conforming basis function. (b) : Diagram for constructing the basis function on $\triangle A B C$.

3.1. Conforming local basis functions on an interface element. The basis functions in the non-conforming IFE space introduced in Section 2 can maintain the jump conditions well, but they may have a small jump along the common edge of two adjacent interface elements within their supports. For example, see Fig. 3.1 (b), the basis function in the non-conforming IFE centered at point $B$ usually is discontinuous at point $D$ where the interface meets with the common edge $\overline{A B}$. Therefore, we would like to find a way to modify the non-conforming local basis functions in interface elements so that the new local basis functions can be pieced together continuously along the common edge of any two interface elements. In addition, the basis functions should still satisfy the jump conditions up to certain accuracy to get sharp solutions for interface problems.

Following the considerations above, we now describe a procedure to construct basis functions in a typical interface element $\triangle A B C$ sketched in Fig. 3.1 (b) such that they can be used to form a conforming IFE space. We assume that the interface meets edges of this element at $D$ and $E$. The key idea is to make sure that some of the local basis functions in two adjacent interface elements, such as $\triangle A B C$ and $\triangle A F B$, can take the same value at the interface point on their common edge, such as point $D$. On the other hand, for a Lagrange type element, the values of a basis function in this element has already had three freedoms at the vertices $A, B$, and $C$. Since we need to form the basis functions together with those in the adjacent interface elements $\triangle A F B$ and $\triangle A C I$ to guarantee the continuity, a basis function in a typical interface element $\triangle A B C$ should have two more freedoms due to the vertices $F$ and $I$.

We use the standard five dimensional Euclidean vector $e_{i}$ (whose $i$-th entry is unity while the other entries are zero) to assign values of a local basis function $\psi_{i}(x, y)$ at the vertices $A, B, C, F$ and $I$, and this basis function is piecewisely constructed as follows:

P1. Use the values at the vertices $A, B, C, F$ and $I$ to form the three non-conforming IFE functions defined on the elements $\triangle A B C, \triangle A F B$ and $\triangle A C I$ respectively.

P2. Assign a value to the point $D$ as the average (or a certain weighted average) of the values at this point of the non-conforming IFE functions defined on the elements $\triangle A B C$ and $\triangle A F B$ formed in $\mathbf{P} 1$.

P3. Similarly, assign a value to the point $E$ as the average (or a certain weighted average) of values at this point of the non-conforming IFE functions defined on the elements $\triangle A B C$ and $\triangle A C I$ formed in $\mathbf{P} 1$. 
P4. Partition the element $\triangle A B C$ into three sub-triangles by an auxiliary line, say line segment $\overline{B E}$, or $\overline{D C}$ such that at least one of acute angles, or the supplementary angle if an angle is bigger than $\pi / 2$, of the triangle formed by the auxiliary line is bigger than or equal to $\pi / 4$.

P5. Define the basis function $\psi_{i}$ to be the piecewise linear function in the three sub-triangles determined by the values at the points $A, B, C, D$ and $E$.

As in Sec. 2, we define a local finite element space on each element $T$ of the partition $\mathcal{T}_{h}$ as follows:

$$
S_{h}(T)=\left\{\begin{array}{l}
\{u(\mathbf{x}) \mid u(\mathbf{x}) \text { is linear on } T\}, \quad \text { if } T \text { is a non-interface element, } \\
\operatorname{span}\left\{\psi_{i}(\mathbf{x}), 1 \leq i \leq 5 \mid \psi_{i}(\mathbf{x}) \text { is defined by P1-P5 }\right\}, \quad \text { otherwise. }
\end{array}\right.
$$

As usual, when $T$ is not an interface element, the dimension of $S_{h}(T)$ is three. However, the dimension of $S_{h}(T)$ is five if $T$ is an interface element, two more freedom are added at the interface points on its edges. We would like to point out that if $T$ is an interface element adjacent to the boundary of $\Omega$, then the dimension of $S_{h}(T)$ becomes four.

REMARK 3.1. Actually $S_{h}(T)$, the local space of shape functions for an interface element is just the five dimensional space of continuous piecewise linear functions on the three subtriangles. The procedure P1-P5 described above defines an interpolation operator from $C^{0}(\Omega)$ to the space spanned by the local shape functions and $S_{h}(\Omega)$ is the image of this interpolation operator.

3.2. A conforming IFE space. For the $i$-th vertex in the partition $\mathcal{T}_{h}$, we let $\phi_{i}(\mathbf{x})$ be the continuous piecewise linear function that satisfies (3.1) and $\left.\phi_{i}\right|_{T} \in S_{h}(T)$ for any element $T \in \mathcal{T}_{h}$. Then we let our new IFE space $S_{h}(\Omega)$ be a set of functions such that

$$
S_{h}(\Omega)=\operatorname{span}\left\{\phi_{i}(\mathbf{x})\right\}
$$

Because of the continuity of its basis functions, this IFE space $S_{h}(\Omega)$ is conforming. Also, this conforming IFE space has the same dimension as the non-conforming IFE space and the standard linear finite element space defined on the partition $\mathcal{T}_{h}$.

The basis function $\phi_{i}$ of $S_{h}(\Omega)$ centered at the $i$-th node has a non-zero support on the six surrounding triangles if the interface does not cut through any of these triangles. This leads to a standard five point stencil $^{4}$. If the coefficient is continuous, i.e. $\rho \equiv 1$, these basis functions become the standard linear basis functions. If the interface cuts through any of the surrounding triangles, then, by the definition of $S_{h}(\Omega)$, the support of this basis function is extended to two more triangles along the direction of the interface, see Fig 3.1 (a), where the support of the basis function includes the triangles marked by dashed lines. As a consequence, the corresponding finite difference scheme will generally have a non-standard nine point stencil. It is worth to point out that the total degree of the freedom of the conforming finite element space is the same as the non-conforming finite element space, and is the same as the standard finite element space using the hat functions.

3.3. Approximation capability of the conforming IFE space. Given a piecewise smooth function $u(x, y)$ that satisfies the jump conditions (1.4)-(1.5) along a smooth interface, we will show that its interpolation function $u_{I}(x, y)$ in the conforming IFE space using the values of $u(x, y)$ at vertices can approximate $u(x, y)$ to second order, and its first derivatives can approximate those of $u(x, y)$ to first order in the maximum norm almost everywhere. We assume that the values of the basis functions at intersections, for example, points $D$ and $E$ in Fig 3.1 (b), are simple averages of the non-conforming interpolation functions in the two neighborhood triangles. From this point of view, the conforming

\footnotetext{
${ }^{4}$ Actually the computation involves a seven point stencil, two of the coefficients are zero due to cancellations.
} 
interpolation function is obtained by perturbing the values of the non-conforming interpolation functions at intersections. From Theorem 2.3, such perturbations are bounded by $C_{1} \tilde{h} h$, where $\tilde{h}$ is the shortest distance from the intersection points, such as $D$ and $E$, to the vertices in interface element, such as $B$ and $A$, in Fig. 3.1 (b). The following lemma shows that the perturbations in the first derivatives between two interpolation functions are of order $h$.

Lemma 3.1. Assume: (i) $T \in \mathcal{T}_{h}$ is an interface element; (ii) $u(x, y)$ is a continuous function that its restriction $u^{i}=\left.u\right|_{T^{i}}$ on $T^{i}, \quad i=+,-$, is twice differentiable in each sub-domain $\Omega^{+} \cap T$ and $\Omega^{-} \cap T$, and satisfies the homogeneous jump conditions (1.4)-(1.5); (iii) $\bar{u}_{I}$ and $u_{I}$ are the interpolation functions of $u$ in the non-conforming and conforming IFE spaces, respectively, then

$$
\begin{aligned}
& \left\|\frac{\partial u_{I}}{\partial x}-\frac{\partial \bar{u}_{I}}{\partial x}\right\|_{\infty, T} \leq(4+\sqrt{2}) C_{1} h, \\
& \left\|\frac{\partial u_{I}}{\partial y}-\frac{\partial \bar{u}_{I}}{\partial y}\right\|_{\infty, T} \leq(4+\sqrt{2}) C_{1} h,
\end{aligned}
$$

where $C_{1}$ is given in Theorem 2.2.

Proof: The results are trivial in every non-interface element. In each interface element, the difference between the two linear interpolation functions are caused by the perturbations of the values at intersections, e.g., points $D$ and $E$ in Fig. 3.1 (b). Since both interpolation functions are linear, we can consider the rate of changes of the first derivatives with respect to the changes in the function values at intersections. We just need to consider one of the sub-triangles within the interface element $\triangle A B C$, see Fig. 3.1 (b), and we distinguish the following cases:

Case 1: The triangle contains one of the angles from the master triangle. In this case, the angle can be $\pi / 4$, as in $\triangle A D E$ and $\triangle B C E$, or $\pi / 2$, as in $\triangle D B C$ if the auxiliary line segment is $\overline{D C}$ instead of $\overline{E B}$. We will only consider the case where the angle is $\pi / 4$ and will use the local coordinates to simplify the proof.

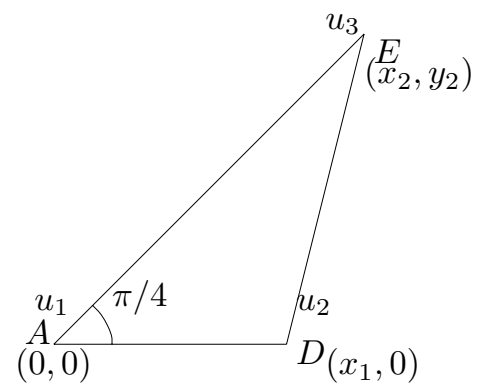

FIG. 3.2. A sub-triangle contains one of angles of the master triangle.

Consider a typical geometry in Fig 3.2, where point $A$ is a vertex, and points $D$ and $E$ are intersections of the interface and the edges of a interface element. The linear function defined in $\triangle A D E$ is

$$
u_{I}(x, y)=u_{1}+\left(u_{2}-u_{1}\right) \frac{x}{x_{1}}+\left(\frac{u_{3}-u_{1}}{x_{2}}-\frac{u_{2}-u_{1}}{x_{1}}\right) y .
$$

Note that $x_{2}=y_{2}$. The partial derivatives with respect to $x$ and $y$ are

$$
\frac{\partial u_{I}}{\partial x}=\frac{u_{2}-u_{1}}{x_{1}}, \quad \frac{\partial u_{I}}{\partial y}=\frac{u_{3}-u_{1}}{x_{2}}-\frac{u_{2}-u_{1}}{x_{1}},
$$


where $u_{1}, u_{2}$, and $u_{3}$ are the interpolation values at points $A, D$, and $E$ respectively. The partial derivatives with respect to $u_{2}$ and $u_{3}$ above are

$$
\begin{gathered}
\frac{\partial}{\partial u_{2}}\left(\frac{\partial u_{I}}{\partial x}\right)=\frac{1}{x_{1}} ; \quad \frac{\partial}{\partial u_{3}}\left(\frac{\partial u_{I}}{\partial x}\right)=0, \\
\frac{\partial}{\partial u_{2}}\left(\frac{\partial u_{I}}{\partial y}\right)=-\frac{1}{x_{1}} ; \quad \frac{\partial}{\partial u_{3}}\left(\frac{\partial u_{I}}{\partial y}\right)=\frac{1}{x_{2}} .
\end{gathered}
$$

Thus

$$
\begin{aligned}
\left|\frac{\partial u_{I}}{\partial x}-\frac{\partial \bar{u}_{I}}{\partial x}\right| & =\left|\frac{\partial}{\partial u_{2}}\left(\frac{\partial u_{I}}{\partial x}\right)\left(u_{I}(D)-\bar{u}_{I}(D)\right)+\frac{\partial}{\partial u_{3}}\left(\frac{\partial u_{I}}{\partial x}\right)\left(u_{I}(E)-\bar{u}_{I}(E)\right)\right| \\
& \leq \frac{1}{\left|x_{1}\right|}\left|u_{I}(D)-\bar{u}_{I}(D)\right| \\
& \leq C_{1} h .
\end{aligned}
$$

The inequality is true within the triangle without second order derivative terms because both $u_{I}$ and $\bar{u}_{I}$ are linear. Similarly

$$
\begin{aligned}
\left|\frac{\partial u_{I}}{\partial y}-\frac{\partial \bar{u}_{I}}{\partial y}\right| & \leq \frac{1}{\left|x_{1}\right|}\left|u_{I}(D)-\bar{u}_{I}(D)\right|+\frac{1}{\left|x_{2}\right|}\left|u_{I}(E)-\bar{u}_{I}(E)\right| \\
& \leq C_{1}(1+\sqrt{2}) h<3 C_{1} h
\end{aligned}
$$

Note that in the proof above we have used the fact that

$$
\begin{aligned}
\left|u_{I}(D)-\bar{u}_{I}(D)\right| & \leq C_{1} h \tilde{h} \leq h\left|x_{1}\right|, \\
\left|u_{I}(E)-\bar{u}_{I}(E)\right| & \leq C_{1} h \tilde{h} \leq h \sqrt{2}\left|x_{2}\right| .
\end{aligned}
$$

Therefore the lemma is true for this case.

If a sub-triangle contains a right angle $\pi / 2$ from the master interface triangle, the proof is similar and we omit the proof to save some space.

Case 2: The interface cuts two right legs of the master triangle. Now we consider a subtriangle in which none of its three angles is from the interface element, for example, $\triangle A D E$ in Fig. 3.3 when $\alpha \geq \pi / 4$. We always choose the triangle that at least one of three acute angles, or the supplementary angle if one is an obtuse, is greater than or equal to $\pi / 4$. If $\alpha<\pi / 4$, then $\alpha^{\prime} \geq \pi / 4$ and we would choose the triangle $\triangle B E D$, which is covered in Case 1 . We proceed with the perturbation analysis on the $\triangle A D E$ for the interpolation function

$$
u_{I}(x, y)=u_{1}+\left(u_{2}-u_{1}\right) \frac{y}{y_{2}}+\left(\frac{u_{3}-u_{1}}{x_{3}}-\frac{\left(u_{2}-u_{1}\right) h}{y_{2} x_{3}}\right) x .
$$

Again $u_{1}, u_{2}$, and $u_{3}$ are the interpolation values at $A, D$, and $E$. The partial derivatives with respect to $x$ and $y$ are

$$
\frac{\partial u_{I}}{\partial x}=\frac{u_{3}-u_{1}}{x_{3}}-\frac{\left(u_{2}-u_{1}\right) h}{y_{2} x_{3}} ; \quad \frac{\partial u_{I}}{\partial y}=\frac{u_{2}-u_{1}}{y_{2}} .
$$

The partial derivatives with respect to $u_{2}$ and $u_{3}$ above are

$$
\begin{gathered}
\frac{\partial}{\partial u_{2}}\left(\frac{\partial u_{I}}{\partial x}\right)=-\frac{h}{y_{2} x_{3}} ; \quad \frac{\partial}{\partial u_{3}}\left(\frac{\partial u_{I}}{\partial x}\right)=\frac{1}{x_{3}}, \\
\frac{\partial}{\partial u_{2}}\left(\frac{\partial u_{I}}{\partial y}\right)=\frac{1}{y_{2}} ; \quad \frac{\partial}{\partial u_{3}}\left(\frac{\partial u_{I}}{\partial y}\right)=0 .
\end{gathered}
$$




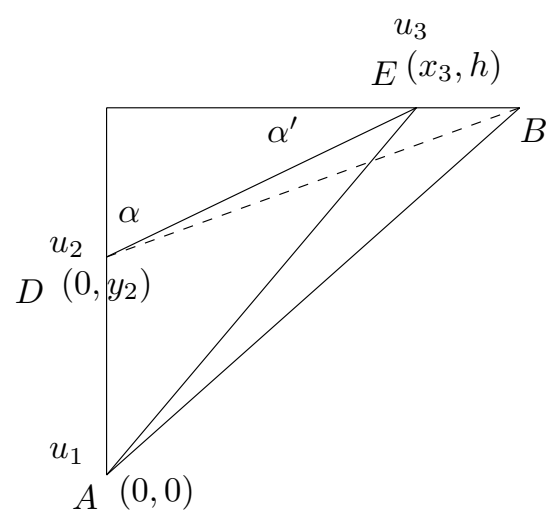

FIG. 3.3. A sub-triangle contains none of the angles of the master triangle, and the interface intersects the two right legs of the master triangle.

Since $\alpha \geq \pi / 4$, we have $x_{3} \geq h-y_{2}$ and

$$
\frac{h}{y_{2} x_{3}} \leq \frac{h}{y_{2}\left(h-y_{2}\right)}
$$

Therefore

$$
\begin{aligned}
\left|\frac{\partial u_{I}}{\partial x}-\frac{\partial \bar{u}_{I}}{\partial x}\right| & \leq \frac{h}{y_{2}\left(h-y_{2}\right)}\left|u_{I}(D)-\bar{u}_{I}(D)\right|+\frac{1}{x_{3}}\left|u_{I}(E)-\bar{u}_{I}(E)\right| \\
& \leq \frac{C_{1} h^{2}}{y_{2}\left(h-y_{2}\right)} \min \left\{y_{2}, h-y_{2}\right\}+C_{1} h \\
& \leq 2 C_{1} h+C_{1} h \\
& \leq 3 C_{1} h .
\end{aligned}
$$

And

$$
\begin{aligned}
\left|\frac{\partial u_{I}}{\partial y}-\frac{\partial \bar{u}}{\partial y}\right| & \leq \frac{1}{y_{2}}\left|u_{I}(D)-\bar{u}_{I}(D)\right| \\
& \leq C_{1} h .
\end{aligned}
$$

Once again we have proved the lemma.

Case 3: The interface cuts one of the right legs and the hypotenuse of a master interface element. A typical picture is shown in Fig. 3.4. One of the angles, $\angle A D E, \angle E D C$, and $\angle D B C$, has to be greater than or equal to $\pi / 4$. Without loss of generality, we assume that $\alpha=\angle A D E \geq \pi / 4$ and the auxiliary line segment is $\overline{D E}$. For other cases, the discussion is similar and is not going to be repeated. We perform the perturbation analysis on the sub-triangle $E D B$. Given the values $\left(u_{1}, u_{2}, u_{3}\right)$ at $B, D$, and $E$, the interpolation function is:

$$
u_{I}(x, y)=u_{1}+\left(u_{2}-u_{1}\right) \frac{y}{y_{2}}+\left(\frac{u_{3}-u_{1}}{x_{3}}-\frac{\left(u_{2}-u_{1}\right)\left(h-x_{3}\right) / y_{2}}{x_{3}}\right) x .
$$

The partial derivatives with respect to $x$ and $y$ are

$$
\frac{\partial u_{I}}{\partial x}=\frac{u_{3}-u_{1}}{x_{3}}-\frac{\left(u_{2}-u_{1}\right)\left(h-x_{3}\right) / y_{2}}{x_{3}} ; \quad \frac{\partial u_{I}}{\partial y}=\frac{u_{2}-u_{1}}{y_{2}} .
$$




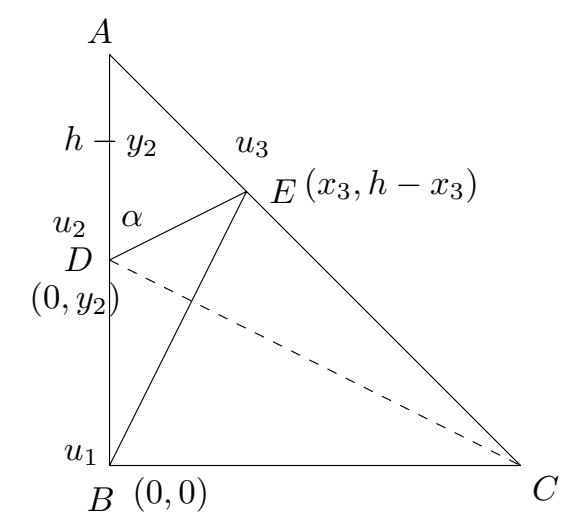

FIG. 3.4. A sub-triangle contains none of the angles of the master triangle, and the interface intersects one of the right legs and the hypotenuse of the master triangle.

The partial derivatives with respect to $u_{2}$ and $u_{3}$ above are

$$
\begin{gathered}
\frac{\partial}{\partial u_{2}}\left(\frac{\partial u_{I}}{\partial x}\right)=-\frac{h-x_{3}}{y_{2} x_{3}} ; \quad \frac{\partial}{\partial u_{3}}\left(\frac{\partial u_{I}}{\partial x}\right)=\frac{1}{x_{3}}, \\
\frac{\partial}{\partial u_{2}}\left(\frac{\partial u_{I}}{\partial y}\right)=\frac{1}{y_{2}} ; \quad \frac{\partial}{\partial u_{3}}\left(\frac{\partial u_{I}}{\partial y}\right)=0 .
\end{gathered}
$$

Since $\alpha \geq \pi / 4$, it is easy to see that $\left(h-y_{2}\right) / 2 \leq x_{3}$ and therefore

$$
\left|\frac{\partial}{\partial u_{2}}\left(\frac{\partial u_{I}}{\partial x}\right)\right| \leq \frac{h-x_{3}}{y_{2} x_{3}} \leq \frac{2 h}{y_{2}\left(h-y_{2}\right)} .
$$

We can see that

$$
x_{3}=\frac{|\overline{A E}|}{\sqrt{2}}
$$

and

$$
\left|\frac{\partial}{\partial u_{3}}\left(\frac{\partial u_{I}}{\partial x}\right)\right| \leq \frac{1}{x_{3}}=\frac{\sqrt{2}}{|\overline{A E}|} .
$$

From the inequalities above, we conclude

$$
\begin{aligned}
\left|\frac{\partial u_{I}}{\partial x}-\frac{\partial \bar{u}_{I}}{\partial x}\right| & \leq \frac{2 h}{y_{2}\left(h-y_{2}\right)}\left|u_{I}(D)-\bar{u}_{I}(D)\right|+\frac{1}{x_{3}}\left|u_{I}(E)-\bar{u}_{I}(E)\right| \\
& \leq 4 C_{1} h+\frac{\sqrt{2}}{|\overline{A E}|}\left|u_{I}(E)-\bar{u}_{I}(E)\right| \\
& \leq C_{1}(4+\sqrt{2}) h .
\end{aligned}
$$

Finally

$$
\begin{aligned}
\left|\frac{\partial u_{I}}{\partial y}-\frac{\partial \bar{u}_{I}}{\partial y}\right| & \leq \frac{1}{y_{2}}\left|u_{I}(D)-\bar{u}_{I}(D)\right| \\
& \leq C_{1} h .
\end{aligned}
$$

Therefore we have proved the inequalities (3.3)-(3.4) are always true.

From the Lemma above, we get the following error estimates for the interpolation function in the conforming IFE space. 
TheOrem 3.2. Let $T \in \mathcal{T}_{h}$ be an interface element, and let $u(x, y)$ be a continuous function whose restriction $u^{i}=\left.u\right|_{T^{i}}$ on $T^{i}, \quad i=+,-$, is twice differentiable in each sub-domain $\Omega^{+} \cap T$ and $\Omega^{-} \cap T$, and satisfies the homogeneous jump conditions (1.4)-(1.5). Then we have the following error estimates

$$
\begin{aligned}
& \left\|\frac{\partial u_{I}}{\partial x}-\frac{\partial u}{\partial x}\right\|_{\infty, T \backslash T_{r}} \leq C_{2} h, \\
& \left\|\frac{\partial u_{I}}{\partial y}-\frac{\partial u}{\partial y}\right\|_{\infty, T \backslash T_{r}} \leq C_{2} h,
\end{aligned}
$$

where

$$
\begin{aligned}
C_{2} & \leq C_{1}(4+\sqrt{2})+\left(22 \rho_{\text {cond }}^{2}+1\right)\left\|\mathcal{D}^{2} u\right\|_{\infty, T} \\
& \leq 7 C_{1}
\end{aligned}
$$

Proof: Denote again the interpolation function using the non-conforming IFE space as $\bar{u}_{I}$, then

$$
\begin{aligned}
\left\|\frac{\partial u_{I}}{\partial x}-\frac{\partial u}{\partial x}\right\|_{\infty, T \backslash T_{r}} & \leq\left\|\frac{\partial u_{I}}{\partial x}-\frac{\partial \bar{u}_{I}}{\partial x}\right\|_{\infty, T \backslash T_{r}}+\left\|\frac{\partial \bar{u}_{I}}{\partial x}-\frac{\partial u}{\partial x}\right\|_{\infty, T \backslash T_{r}} \\
& \leq C_{1}(4+\sqrt{2}) h+\left(22 \rho_{\text {cond }}^{2}+1\right)\left\|\mathcal{D}^{2} u\right\|_{\infty, T} h,
\end{aligned}
$$

from Lemma 3.1 and Theorem 2.2, respectively. Similar proof can be carry out for $\partial u_{I} / \partial y$.

From this theorem and the proof of Theorem 2.3, we have the following theorem for the error estimate of the interpolation function.

TheOrem 3.3. Assume: (i) $T \in \mathcal{T}_{h}$ is an interface element; (ii) $u(x, y)$ is a continuous function that its restriction $u^{i}=\left.u\right|_{T^{i}}$ on $T^{i}, \quad i=+,-$, is twice differentiable in each sub-domain $\Omega^{+} \cap T$ and $\Omega^{-} \cap T$, and satisfies the homogeneous jump conditions (1.4)-(1.5); (iii) $u_{I}$ is the interpolation function of $u$ in the conforming IFE spaces. Then the following inequality holds

$$
\left|u(\mathbf{x})-u_{I}(\mathbf{x})\right| \leq \begin{cases}C_{3} \tilde{h} h, & \text { if } \mathbf{x} \in T \backslash T_{r} \\ C_{4} h^{2}, & \text { if } \mathbf{x} \in T_{r}\end{cases}
$$

where $C_{4}$ is a constant, $\tilde{h}$ is the shortest distance between $\mathbf{x}$ and the vertices of $T$ that are on the same side of the interface as $\mathbf{x}$, and

$$
C_{3} \leq C_{2}+\left\|\mathcal{D}^{2} u\right\|_{\infty, T}
$$

\section{REMARK 3.2.}

- The interpolation errors actually depend on the jump in the coefficient, the spatial step size h, and the geometry. The error generally is not a monotonous function of $h$ because the error depends on the relative position of the interface and the underlying grid, see [31].

- One may try to set the intersections between the interface and edges as new nodal points and then to use the standard piecewise linear finite element space. The problem is that there may be skinny triangles that will deteriorate the convergence rate. However, we know that the solution to the interface problem is not independent across the interface. In our approach, we can use one of the nice triangles to approximate the partial derivatives, and then pass the information to the skinny ones, if there are any, using the jump conditions as in the proof process for Lemma 3.1. 
We now define the conforming IFE solution to the interface problem as a function $u_{h} \in S_{h 0}(\Omega)$ such that

$$
\iint_{\Omega} \beta(x, y) \nabla u_{h} \nabla v_{h} d x d y=\iint_{\Omega} f v_{h} d x d y, \text { for all } v_{h} \in S_{h 0}(\Omega),
$$

and again, we let $S_{h 0}(\Omega)=\left\{\phi \in S_{h}(\Omega)|\phi|_{\partial \Omega}=0\right\}$. For a non-homogeneous flux condition, the contribution again is a line integral in the weak form.

For this conforming IFE solution, we can obtain an error estimate in the energy norm given in the following theorem.

THEOREM 3.4. Let $u$ be the solution of (1.6) with $Q \equiv 0$, and $u_{h}$ be the conforming IFE solution. If $u$ is in $H_{0}^{1}(\Omega)$ and is piecewise twice differentiable on each sub-domain $\Omega^{i}, i=+$ and $i=-$, then we have the following error estimate:

$$
\left\|u-u_{h}\right\|_{1, \Omega} \leq C_{5} h
$$

where $C_{5}$ is a constant independent of $h$.

Proof: Since $u, u_{h}$, and the IFE finite dimensional space, all belong to $H_{0}^{1}(\Omega)$. From the standard FEM theory, $u_{h}$ is the best solution in the IFE space in the $H^{1}$ norm. Therefore we have

$$
\begin{aligned}
\left\|u-u_{h}\right\|_{1, \Omega} & \leq \tilde{C}\left\|u-u_{I}\right\|_{1, \Omega} \\
& \leq \tilde{C}\left(\left\|u-u_{I}\right\|_{1, \sum T \backslash T_{r}}+\left\|u-u_{I}\right\|_{1, \sum T_{r}}\right)
\end{aligned}
$$

where $u_{I} \in H^{1}$ is the interpolation function of $u$ in the conforming IFE space. $\sum T \backslash T_{r}$ is the union of the mis-matched region of the line segments and the interface as shown in Fig. 2.1. From Theorem 3.2, we know that $u-u_{I}$ and its first derivatives are of $O\left(h^{2}\right)$ and $O(h)$, respectively, in the maximum norm on $T \backslash T_{r}$ of an element $T$, therefore, $u-u_{I}$ should be of $O(h)$ in the $H^{1}$ norm on the unions of these regions as well. On each $T_{r}, u-u_{I}$ and its first derivatives are of order $O\left(h^{2}\right)$ and $O(1)$. However, with the interface being approximated by the line segment on each element, the area of each $T_{r}$ is order of $O\left(h^{3}\right)$. Since the interface is one dimensional lower than the solution domain, we also conclude that

$$
\left\|u-u_{I}\right\|_{1, \sum T_{r}} \approx h
$$

which leads to the result of this theorem.

\section{REMARK 3.3.}

- The finite element solution $u(x)$ in the conforming IFE space belongs to $H^{1}(\Omega)$ but generally is not in $H^{2}(\Omega)$ if $\beta(x, y)$ has a discontinuity across the interface, see Fig. 3.5. This is the main reason that the standard finite element does not work well.

- For many practical interface problems, the solutions are indeed piecewise smooth. Generally, if the source term $f(x, y) \in L^{2}(\Omega)$ is also $\gamma^{\text {th }}$-Hölder piecewise continuous for $\gamma>0$, then the solution $u(x, y)$ is piecewise twice differentiable, see [12].

- We believe that piecewise smooth requirement of the solution in the theorem can be relaxed to piecewise $H^{2}\left(\Omega^{i}\right)$ by developing corresponding interpolation theory in the Sobolev space. Such investigation is not straightforward and is under way. Once such theory is established, we believe that the second order or nearly second order accuracy in the maximum norm can also be proved.

3.4. A numerical example for the conforming IFE method. We present some numerical results for the same boundary value problem as in Sec. 2.5. We also report the error of the interpolation 


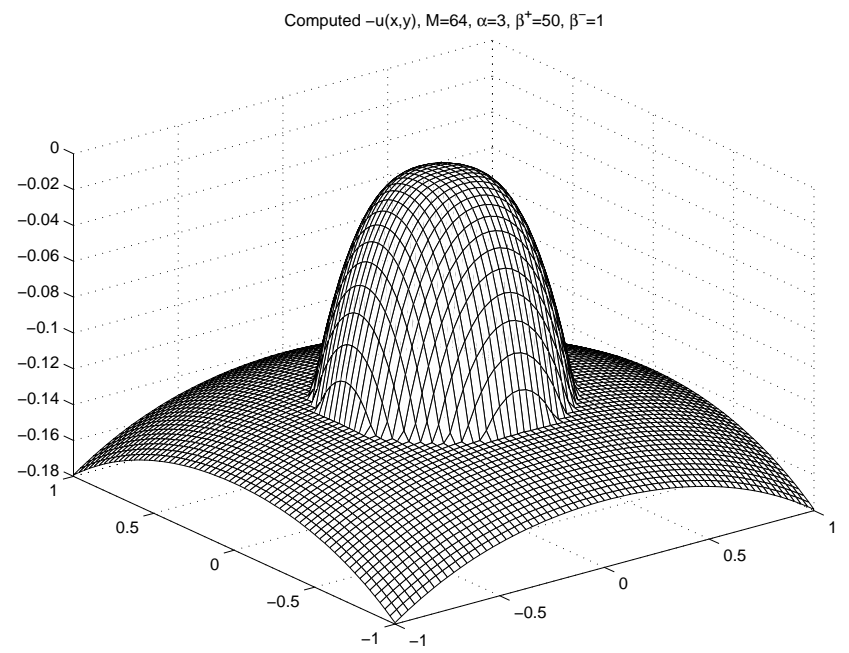

FIG. 3.5. Plot of the computed solution with $M=64, \alpha=3, \beta^{+}=50, \beta^{-}=1$. The parameters are chosen to illustrate the fact that the solution $u(x, y)$ belongs to $H_{0}^{1}(\Omega)$ but is not in $H_{0}^{2}(\Omega)$. However, the solution is piecewise smooth.

(a)

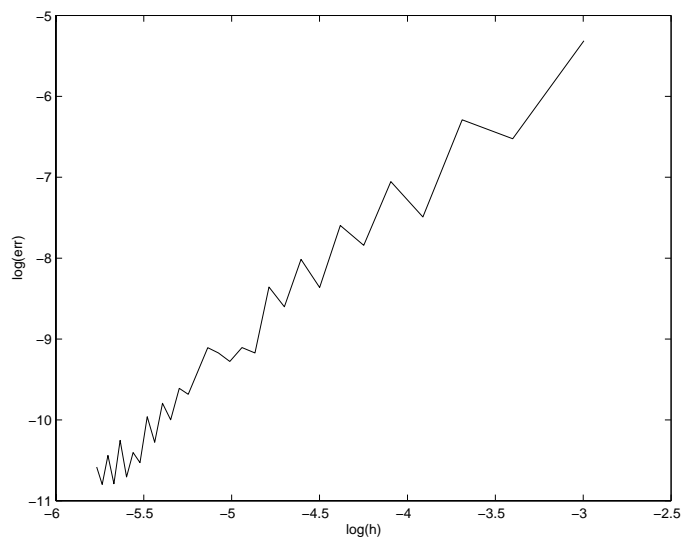

(b)

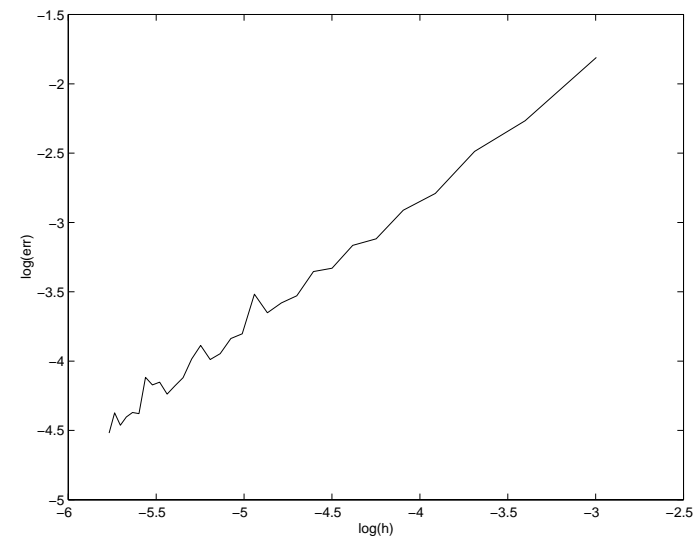

FIG. 3.6. The interpolation errors in the maximum norm versus the mesh size $h$ for conforming basis functions in $\log -\log$ scale with $r_{0}=\pi / 6.28, \beta^{-}=1$, and $\beta^{+}=1000 . \quad(a)$ : The linear regression analysis gives $\left\|u-u_{I}\right\|_{\infty} \approx$ $3.22816 h^{2.06743} ;(b):$ The error in the partial derivative $\partial u / \partial x$ excluding the region $\sum T_{r}$. The linear regression analysis gives $\left\|\left(u-u_{I}\right)_{x}\right\|_{\infty, \sum T \backslash T_{r}} \approx 2.89806 h^{0.96056}$.

function that is very important for the finite element theory, and is useful in deriving the error estimate for the maximum norm.

Fig. 3.6 (a) plots the errors between the exact solution and its interpolation functions in the conforming IFE space $S_{h}(\Omega)$ with the jump ratio $\rho=\beta^{-} / \beta^{+}=1: 1000$ and various partition size $h$. Fig. 3.6 (b) is the plot of the error in the $x$ partial derivative of the interpolation function. We obtained similar result with other ratios and partial derivatives. Thus, this example confirmed our error analysis for the interpolation function. Note that the magnitude of the interpolation error is about $10^{-4}$ for the solution and $10^{-2}$ for the $x$ partial derivate in a typical 160 by 160 grid.

Fig. 3.7 plots the errors in the maximum norm of the conforming IFE solutions $u_{h}$ from $S_{h}(\Omega)$ with various $h$ for two different ratios. The linear regression analysis shows that data in Fig. 3.7 obey

$$
\left\|u-u_{h}\right\|_{\infty} \approx 6.85126 h^{2.01002}, \quad \rho=1: 1000
$$


(a)

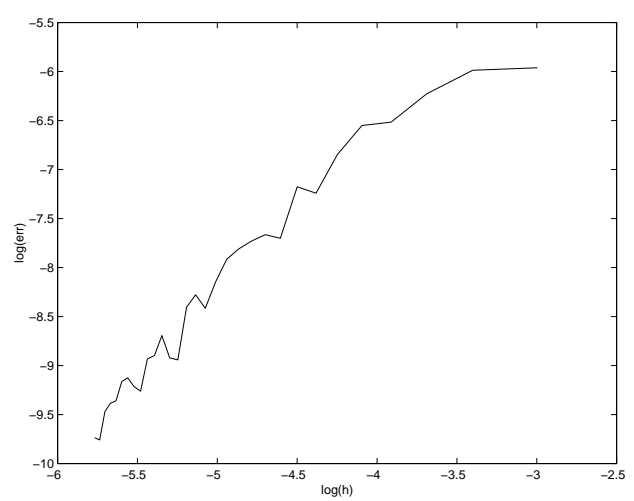

(b)

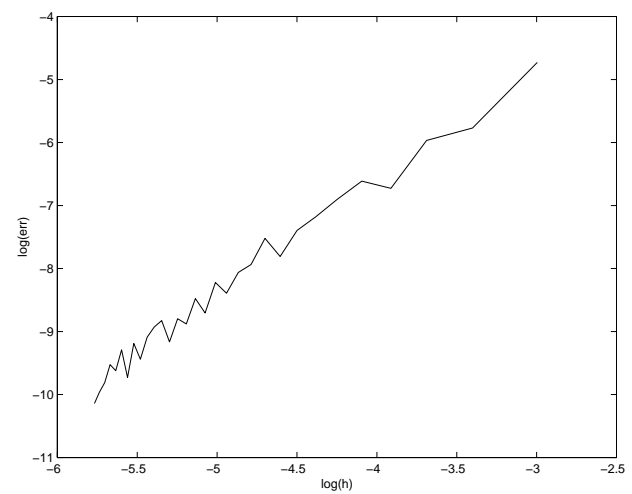

FIG. 3.7. Errors of finite element solutions obtained from the conforming basis function in the maximum norm versus the mesh size $h$ in $\log -\log$ scale with $r_{0}=\pi / 6.28$. (a): $\beta^{-}=1, \beta^{+}=1000$. The linear regression analysis gives $\left\|u-u_{h}\right\|_{\infty} \approx 6.85126 h^{2.01002} ;(b): \beta^{-}=1000, \beta^{-}=1$. The linear regression analysis gives $\left\|u-u_{h}\right\|_{\infty} \approx 5.65703 h^{2.01542}$.

$$
\left\|u-u_{h}\right\|_{\infty} \approx 5.65703 h^{2.01542}, \quad \rho=1000: 1,
$$

which suggest that the conforming IFE finite element solution has a second order convergence rate in the maximum norm.

3.4.1. A comparison with the FEM method with added nodes. As suggested by several colleagues and other investigators (including one of the referee of this paper), we have tested a slightly different method. We consider the finite element method based on a triangulation in which most of the triangles are uniform right triangles from a Cartesian grid. In the neighborhood of the interface, we add some new nodal points at the intersections of the interface and the edges of the uniform Cartesian right triangles. Specifically, this triangulation is generated as follows:

1. We first generate a Cartesian triangulation composed of the right triangles over $\Omega$.

2. We keep all the elements over the non-interface triangles unchanged.

3. For each interface triangle, we break it into three small triangles in the same way as we did in step $\mathbf{P} 4$ in Section 3.1, see also Fig.3.1. Therefore the break-up satisfies the same maximum angle condition as we did earlier for our conforming IFE method.

The standard Galerkin finite element method with the usual linear basis functions is then applied to this triangulation. We will call this method the finite element method using a Cartesian grid with added nodes, or FEMCGAN, for short. The computational complexity of this approach is about the same as our conforming finite element method. Both methods are new, some futures of them are summarized below:

- The convergence result of Theorem 3.4 is also valid for the FEMCGAN method. However, this is guaranteed only with the choice of the maximum angles proposed in this paper.

- In the FEMCGAN approach, all the intersections between the interface and the edges of Cartesian triangles are the added nodal points. However, in our IFE methods, either non-conforming or conforming, those intersections are not part of the nodal points. Therefore, the linear system of equation from our approach will be order $O(1 / h)$ smaller compared with that from the FEMCGAN approach. More importantly, many linear solvers based on Cartesian grids such as MGD9V [10] can be applied to our non-conforming or conforming IFE methods but not to the FEMCGAN approach. In many applications, we are only interested in the solution at the grid points, there is no need to recover the solution at the points of the intersections. The Cartesian 
grid methods of this paper are developed for this purpose.

- The FEMCGAN space contains the IFE space, so we can expect the energy norm of the error is smaller than that obtained from the IFE method, see Table 3.1.

- The FEMCGAN method is a little bit easier to implement compared with the conforming IFE method.

In Table 3.1, we show the results of the errors in $L^{2}(\Omega)$ and energy norms of the FEMCGAN approach and the IFE method. The problem set-up is the same as the example in Section 2.5 and in Section 3.4. The ratio in the table is the ratio of two consecutive errors. If the error is proportional to $h$, then the ratio should approach number two. If the error is proportional to $h^{2}$, then the ratio should approach number four. We can see clearly from the table that the two new methods are comparable. Both methods give second order accurate results in the $L^{2}(\Omega)$ norm, first order accuracy in the energy norm.

\begin{tabular}{||c|c|c|c|c||}
\hline \hline & \multicolumn{5}{|c||}{ The FEMCGAN method } \\
\hline$h$ & $e_{0}(h)$ & ratio & $e_{a}(h)$ & ratio \\
\hline $1 / 20$ & $5.5479 \times 10^{-4}$ & & $3.0085 \times 10^{-2}$ & \\
\hline $1 / 40$ & $1.4040 \times 10^{-4}$ & 3.9516 & $1.5376 \times 10^{-2}$ & 1.9566 \\
\hline $1 / 80$ & $3.5525 \times 10^{-5}$ & 3.9520 & $7.7803 \times 10^{-3}$ & 1.9762 \\
\hline $1 / 160$ & $9.1518 \times 10^{-6}$ & 3.8817 & $3.9160 \times 10^{-3}$ & 1.9868 \\
\hline \hline \multicolumn{5}{|c|}{ The conforming IFE method } \\
\hline $1 / 20$ & $7.7184 \times 10^{-4}$ & & $3.4742 \times 10^{-2}$ & \\
\hline $1 / 40$ & $1.9050 \times 10^{-4}$ & 4.0516 & $1.7136 \times 10^{-2}$ & 2.0275 \\
\hline $1 / 80$ & $4.5729 \times 10^{-5}$ & 4.1659 & $8.4975 \times 10^{-3}$ & 2.0165 \\
\hline $1 / 160$ & $1.0596 \times 10^{-5}$ & 4.3158 & $4.1195 \times 10^{-3}$ & 2.0627 \\
\hline \hline
\end{tabular}

TABLE 3.1

Comparisons of errors of the FEMCGAN and the IFE methods, where $e_{0}(h)$ and $e_{a}(h)$ are errors of a numerical solution in the $L^{2}(\Omega)$ and energy norms respectively. The example is the same as the example in Section 2.5 for the case when $\beta^{-}=1, \beta^{+}=1000$.

The grid refinement analysis from a few selected grids does not provide a good indication for the convergence in the $L^{\infty}$ norm because the errors in the $L^{\infty}$ norm computed from both methods oscillate as explained in [31]. Therefore, we use the linear regression analysis to present the convergence in $L^{\infty}$ norm for both methods with 10 grid increment. For the FEMCGAN method, we have following results:

$$
\begin{aligned}
& \text { error in } L^{2} \text { norm } \approx 0.20822 h^{1.98032}, \\
& \text { error in } H^{1} \text { norm } \approx 0.58835 h^{0.99016}, \\
& \text { error in energy norm } \approx 0.60372 h^{0.99231}, \\
& \text { error in } L^{\infty} \text { norm } \approx 0.14247 h^{1.85615},
\end{aligned}
$$

and for the conforming IFE method, the results are

$$
\begin{aligned}
& \text { error in } L^{2} \text { norm } \approx 0.77413 h^{2.21055}, \\
& \text { error in } H^{1} \text { norm } \approx 0.66915 h^{1.01420},
\end{aligned}
$$




$$
\begin{aligned}
& \text { error in energy norm } \approx 0.92382 h^{1.06659}, \\
& \text { error in } L^{\infty} \text { norm } \approx 1.70096 h^{2.01002} .
\end{aligned}
$$

Again, these numerical results indicate that these two methods perform comparably.

4. Conclusions. In this paper, we have developed two immersed finite element (IFE) spaces for interface problems using Cartesian grids. Error estimates are obtained for the interpolation functions in both conforming and non-conforming new finite element spaces. The non-conforming IFE method behaviors better than that of the standard linear finite element solutions because the method produces sharp solutions near or on the interfaces. In addition, this method is very simple, and can be extended to three dimensions easily. By extending the support of some basis functions, we are able to construct a conforming IFE space. The Galerkin finite element method based on the conforming IFE space has been proved to be convergent for piecewise solutions that may not be in $H^{2}(\Omega)$. A modification to the conforming finite elements with added nodes near the interface is also discussed and tested.

The ideas of this paper can be modified for almost any arbitrary grids that are not necessarily aligned with interfaces. The methods based on the Cartesian grids can be easily used as finite difference methods. Thus they can be incorporated into other Cartesian grids based methods and packages, for examples, LeVeque's Clawpack and Berger's AMR package, to solve interface problems.

5. Acknowledgments. In this research, Zhilin Li is supported in part by USA ARO grants, 39676MA and 43751-MA, USA NSF grants DMS-0073403 and DMS-0201094; USA North Carolina State University FR\&PD grant; Tao Lin is supported in part a USA NSF grant DMS-97-04621. Special thanks to Thomas Hou for his participation and contribution to this project. We are also grateful to R. LeVeque, K. Bube, and T. Chan for useful discussions.

\section{REFERENCES}

[1] L. M. Adams. A multigrid algorithm for immersed interface problems. pages 1-14. Proceedings of Copper Mountain Multigrid Conference, NASA Conference Publication 3339, 1995.

[2] I. Babuška. The finite element method for elliptic equations with discontinuous coefficients. Computing, 5:207-213, 1970.

[3] J. B. Bell, C. N. Dawson, and G. R. Shubin. An unsplit, higher order Godunov method for scalar conservation laws in multiple dimensions. J. Comput. Phys., 74:1-24, 1988.

[4] J. B. Bell and D. L. Marcus. A second-order projection method for variable-density flows. J. Comput. Phys., 101:334348, 1992.

[5] R. P. Beyer. A computational model of the cochlea using the immersed boundary method. J. Comput. Phys., 98:145-162, 1992.

[6] J. Bramble and J. King. A finite element method for interface problems in domains with smooth boundaries and interfaces. Advances in Comput. Math., 6:109-138, 1996.

[7] Z. Chen and J. Zou. Finite element methods and their convergence for elliptic and parabolic interface problems. Numer. Math., 79:175-202, 1998.

[8] A. J. Chorin. Numerical solution of the Navier-Stokes equations. Math. Comp., 22:745-762, 1968.

[9] P. G. Ciarlet. The finite element method for elliptic problems. North Holland, 1978.

[10] D. De Zeeuw. Matrix-dependent prolongations and restrictions in a blackbox multigrid solver. J. Comput. Appl. Math., 33:1-27, 1990.

[11] B. Engquist and H.K. Zhao. Absorbing boundary conditions for domain decomposition. Applied Numerical Mathematics, 27:341-365, 1998.

[12] L. C. Evans. Partial Differential Equations. AMS, 1998.

[13] L. J. Fauci. Interaction of oscillating filaments - A computational study. J. Comput. Phys., 86:294-313, 1990.

[14] A. L. Fogelson. A mathematical model and numerical method for studying platelet adhesion and aggregation during blood clotting. J. Comput. Phys., 56:111-134, 1984. 
[15] A. L. Fogelson and J. P. Keener. Immersed interface methods for Neumann and related problems in two and three dimensions. SIAM J. Sci. Comput., 22:1630-1684, 2000.

[16] A. L. Fogelson and C. S. Peskin. Numerical solution of the three dimensional Stokes equations in the presence of suspended particles. In Proc. SIAM Conf. Multi-phase Flow. SIAM, June 1986.

[17] L. Greengard and M. Moura. On the numerical evaluation of electrostatic fields in composite materials. Acta Numerica, pages 379-410, 1994.

[18] H. Han. The numerical solutions of the interface problems by infinite element methods. Numer. Math., 39:39-50, 1982.

[19] T. Hou, Z. Li, S. Osher, and H. Zhao. A hybrid method for moving interface problems with application to the Hele-Shaw flow. J. Comput. Phys., 134:236-252, 1997.

[20] T. Y. Hou, J. S. Lowengrub, and M. J. Shelley. Removing the stiffness from interfacial flows with surface tension. J. Comput. Phys., 114:312-338, 1994.

[21] T.Y. Hou and B.T.R. Wetton. Second order convergence of a projection scheme for the incompressible Navier-Stokes equations with boundaries. SIAM J. Numer. Anal., 30:609-629, 1993.

[22] H. Huang and Z. Li. Convergence analysis of the immersed interface method. IMA J. Numer. Anal., 19:583-608, 1999.

[23] C. Johnson. Numerical solution of Partial Differential Equations by the Finite Element Method. Cambridge University Press, 1987.

[24] R. J. LeVeque. Clawpack and Amrclaw - Software for high-resolution Godunov methods. 4-th Intl. Conf. on Wave Propagation, Golden, Colorado, 1998.

[25] R. J. LeVeque and Z. Li. The immersed interface method for elliptic equations with discontinuous coefficients and singular sources. SIAM J. Numer. Anal., 31:1019-1044, 1994.

[26] R. J. LeVeque and Z. Li. Immersed interface method for Stokes flow with elastic boundaries or surface tension. SIAM J. Sci. Comput., 18:709-735, 1997.

[27] R. J. LeVeque and C. Zhang. Immersed interface methods for wave equations with discontinuous coefficients. Wave Motion, 25:237-263, 1997.

[28] Z. Li. The Immersed Interface Method - A Numerical Approach for Partial Differential Equations with Interfaces. $\mathrm{PhD}$ thesis, University of Washington, 1994.

[29] Z. Li. A note on immersed interface methods for three dimensional elliptic equations. Computers Math. Appl., 31:9-17, 1996.

[30] Z. Li. Immersed interface method for moving interface problems. Numerical Algorithms, 14:269-293, 1997.

[31] Z. Li. A fast iterative algorithm for elliptic interface problems. SIAM J. Numer. Anal., 35:230-254, 1998.

[32] Z. Li, D. McTigue, and J. Heine. A numerical method for diffusive transport with moving boundaries and discontinuous material properties. International J. Numer. \& Anal. Method in Geomechanics, 21:653-662, 1997.

[33] Z. Li and B. Soni. Fast and accurate numerical approaches for Stefan problems and crystal growth. Numerical Heat Transfer, B: Fundamentals, 35:461-484, 1999.

[34] Z. Li and J. Zou. Theoretical and numerical analysis on a thermo-elastic system with discontinuities. J. of Comput. Appl. Math., 91:1-22, 1998.

[35] A. Mayo. The fast solution of Poisson's and the biharmonic equations on irregular regions. SIAM J. Numer. Anal., 21:285-299, 1984.

[36] A. Mayo and A. Greenbaum. Fast parallel iterative solution of Poisson's and the biharmonic equations on irregular regions. SIAM J. Sci. Stat. Comput., 13:101-118, 1992.

[37] A. McKenney, L. Greengard, and Anita Mayo. A fast poisson solver for complex geometries. J. Comput. Phys., 118, 1995.

[38] R. H. Nochetto, M. Paolini, and C. Verdi. An adaptive finite element method for two-phase stefan problems in two space dimensions. SIAM J. Sci. Stat. Comput., 12:1207-1244, 1991.

[39] S. Osher and J. A. Sethian. Fronts propagating with curvature-dependent speed: Algorithms based on Hamilton-Jacobi formulations. J. Comput. Phys., 79:12-49, 1988.

[40] C. S. Peskin. Numerical analysis of blood flow in the heart. J. Comput. Phys., 25:220-252, 1977.

[41] C. S. Peskin. Lectures on mathematical aspects of physiology. Lectures in Appl. Math., 19:69-107, 1981.

[42] G. R. Shubin and J. B. Bell. An analysis of the grid orientation effect in numerical simulation of miscible displacement. Comp. Meth. Appl. Mech. Eng., 47:47-71, 1984.

[43] D. Sulsky and J. U. Brackbill. A numerical method for suspension flow. J. Comput. Phys., 96:339-368, 1991.

[44] M. Sussman, P. Smereka, and S. Osher. A level set approach for computing solutions to incompressible two-phase flow. J. Comput. Phys., 114:146-159, 1994.

[45] A. Wiegmann. The explicit jump immersed interface method and interface problems for differential equations. PhD thesis, University of Washington, 1998. 
[46] A. Wiegmann and K. Bube. The immersed interface method for nonlinear differential equations with discontinuous coefficients and singular sources. SIAM J. Numer. Anal., 35:177-200, 1998.

[47] J. Xu. Error estimates of the finite element method for the 2nd order elliptic equations with discontinuous coefficients. J. Xiangtan University, No. 1:1-5, 1982. 